\title{
Sylviculture d'adaptation aux changements climatiques : des concepts à la réalité Compte-rendu d'un colloque tenu au Carrefour Forêts 2019
}

\author{
par Nelson Thiffault ${ }^{1}$, $^{*}$, Patricia Raymond ${ }^{3}$, Jean-Martin Lussier ${ }^{1}$, Isabelle Aubin ${ }^{2,4}$, Samuel Royer-Tardif4, \\ Anthony W. D'Amato ${ }^{5}$, Frédérik Doyon ${ }^{2,6}$, Benoit Lafleur ${ }^{2,7}$, Martin Perron ${ }^{3,8}$, Jean Bousquet ${ }^{8}$, Nathalie Isabel ${ }^{9,10}$, \\ Sylvie Carles ${ }^{11}$, Patrick Lupien ${ }^{12}$ et Annie Malenfant ${ }^{13}$
}

\begin{abstract}
RÉSUMÉ
Dans le cadre du Carrefour Forêts 2019, le colloque Sylviculture d'adaptation aux changements climatiques : des concepts à la réalité, organisé conjointement par la Direction de la recherche forestière du ministère des Forêts, de la Faune et des Parcs du Québec et le Centre canadien sur la fibre de bois de Ressources naturelles Canada, a rassemblé une centaine d'acteurs du milieu forestier incluant des ingénieurs forestiers, des biologistes, des techniciens forestiers, des propriétaires de boisés, des professeurs, des chercheurs et des étudiants. Des conférenciers de différentes régions du Québec et du Vermont ont partagé les connaissances actuelles en rapport avec la sylviculture d'adaptation afin d'amorcer la réflexion et le dialogue quant aux actions pouvant être entreprises dès maintenant pour favoriser l'adaptation des forêts aux changements anticipés. Les présentations ont abordé les fondements théoriques de la sylviculture d'adaptation, pour ensuite présenter des réflexions ou des études de cas portant sur différents types d'écosystèmes, des outils innovants ou des initiatives de recherche. Le colloque a également laissé une place aux praticiens qui ont pu décrire les défis et les occasions qu'offre la sylviculture d'adaptation dans leur pratique professionnelle. Au terme d'une activité interactive, les participants ont ciblé les sécheresses accrues, les coupes partielles et les plantations mélangées comme des thèmes importants qui devraient animer les échanges entre les chercheurs et les praticiens dans les années à venir.
\end{abstract}

Mots-clés: traitements sylvicoles, changements globaux, vulnérabilité, sécheresse, forêt décidue, forêt mixte, forêt boréale, aménagement écosystémique, amélioration génétique, génomique, mise en œuvre, écologie fonctionnelle

\begin{abstract}
As part of the Carrefour Forêts 2019 Conference, the forest research branch of Quebec's Ministère des Forêts, de la Faune et des Parcs and the Canadian Wood Fibre Centre of Natural Resources Canada organized jointly the symposium "Adaptive silviculture to climate change: from concepts to reality". The symposium brought together a hundred actors from the forest sector including engineers, biologists, technicians, private woodlot owners, professors, researchers and students. Speakers from different regions of Quebec and Vermont shared current knowledge related to adaptive silviculture in order to nurture reflexion and dialogue about actions that can be taken today to help forests to adapt with the anticipated changes. The symposium covered the theoretical framework of adaptive silviculture, then offered case studies of different ecosystems, innovative tools or research initiatives. The symposium also included presentations by practitioners who described the challenges and opportunities offered by adaptive silviculture in their professional practice. Based on an interactive discussion, participants identified increased drought events, partial cuttings and mixed-species plantations as important topics on which researchers and practitioners should interact in years to come.
\end{abstract}

Keywords: silvicultural treatments, global change, vulnerability, drought, hardwood forests, mixedwood forests, boreal forests, ecosystem-based management, tree breeding, genomics, implementation, functional ecology

\footnotetext{
${ }^{1}$ Centre canadien sur la fibre de bois, Service canadien des forêts, Ressources naturelles Canada, 1055, rue du P.E.P.S., C.P. 10380, succ. Sainte-Foy, Québec (QC) G1V 4C7, Canada; *Auteur de correspondance. Courriel : nelson.thiffault@canada.ca

${ }^{2}$ Centre d'étude de la forêt, Faculté de foresterie, de géographie et de géomatique, Université Laval, Québec (QC) G1V 0A6, Canada

${ }^{3}$ Direction de la recherche forestière, Ministère des Forêts, de la Faune et des Parcs du Québec, 2700, rue Einstein, Québec (QC) G1P 3W8, Canada

${ }^{4}$ Centre de foresterie des grands Lacs, Service canadien des forêts, Ressources naturelles Canada, 1219, Queen St. E., Sault Ste. Marie (ON) P6A 2E5, Canada

${ }^{5}$ University of Vermont, Rubenstein School of Environment and Natural Resources, 204E, Aiken Center, Burlington (VT) 05405, USA

${ }^{6}$ Université du Québec en Outaouais, Institut des sciences de la forêt tempérée, 58, rue Principale, Ripon (QC) J0V 1V0, Canada

${ }^{7}$ Université du Québec en Abitibi-Témiscamingue, Institut de recherche sur les forêts, 445, boul. de l'Université, Rouyn-Noranda (QC) J9X 5E4, Canada

${ }^{8}$ Chaire de recherche du Canada en génomique forestière, Université Laval, Faculté de foresterie, de géographie et de géomatique, Université Laval, 1030, avenue de la Médecine, Québec (QC) G1V 0A6, Canada

${ }^{9}$ Département des sciences du bois et de la forêt, Faculté de foresterie, de géographie et de géomatique, Université Laval, 2405, rue de la Terrasse, Québec (QC) G1V 0A6, Canada

${ }^{10}$ Centre de foresterie des Laurentides, Service canadien des forêts, Ressources naturelles Canada, 1055, rue du P.E.P.S., C.P. 10380, succ. Sainte-Foy, Québec (QC) G1V 4C7, Canada

${ }^{11}$ Direction générale de la production de semences et de plants forestiers, Ministère des Forêts, de la Faune et des Parcs, 5700, 4e Avenue Ouest, Québec (QC) G1H 6R1, Canada

${ }^{12}$ Syndicat des producteurs de bois de la Mauricie, Lanaudière et du Centre-du-Québec, 2410, rue de l'Industrie, Trois-Rivières (QC) G8Z 4R5, Canada

${ }^{13}$ Direction de la gestion des forêts de la Gaspésie-îles-de-la-Madeleine, Ministère des Forêts, de la Faune et des Parcs, 195, boul. Perron Est, Caplan (QC) G0C 1H0, Canada
} 


\section{Introduction}

Les changements climatiques observés et à venir induiront des stress importants sur les forêts. Bien que plusieurs de ces effets demeurent incertains (Millar et al. 2007; Bonan 2008; Gauthier et al. 2015), il semble acquis qu'ils s'intensifieront au cours des décennies à venir (Price et al. 2013). Par exemple, les cas de dépérissement des forêts deviendront possiblement plus fréquents (Allen et al. 2010), avec des résultats sur les interactions entre les activités forestières, le couvert forestier, le sol et les ravageurs (Trumbore et al. 2015). Les changements climatiques ont également des effets intangibles, mais bien réels sur les activités d'aménagement forestier, tels que l'incertitude qu'ils génèrent et que doivent gérer les aménagistes et les sylviculteurs (Klenk et al. 2011; Williamson et al. 2019).

La sylviculture offre le potentiel d'améliorer la capacité d'adaptation des forêts aux effets des changements climatiques (Nagel et al. 2017). La sylviculture d'adaptation s'inscrit ainsi comme un outil important pour réaliser l'aménagement durable des forêts du Québec. À ce titre, plusieurs stratégies peuvent être explorées, notamment pour favoriser la résistance, la résilience ou la transition des peuplements en se basant sur la compréhension de leur dynamique naturelle (Millar et al. 2007). Alors que les chercheurs explorent le cadre théorique, le potentiel et les limites de ces différentes stratégies, les aménagistes et les sylviculteurs relèvent le défi quotidien d'intégrer ces nouvelles connaissances dans leur pratique professionnelle. Or, l'intégration des plus récents développements scientifiques dans la pratique n'est pas simple (Böcher et Krott 2014).

Dans cette optique, le Carrefour Forêts 2019 organisé par le ministère des Forêts, de la Faune et des Parcs du Québec (MFFP) a réuni plus de 1700 participants provenant des sphères scientifiques, pratiques et politiques de la foresterie québécoise afin de favoriser les échanges entre chercheurs et praticiens (Ministère des Forêts de la Faune et des Parcs 2019). Dans son ensemble, le Carrefour a couvert les thèmes de la dynamique naturelle des écosystèmes, de l'aménagement écosystémique et la sylviculture, des changements climatiques, de l'intégration des enjeux sociaux et environnementaux dans la gestion forestière, des aspects économiques de l'aménagement forestier en forêt publique et en forêt privée, de même que de l'innovation et du développement dans l'industrie des produits forestiers. Ces différents thèmes ont été abordés lors de colloques, d'ateliers, de formations et d'une visite sur le terrain.

Dans le cadre du Carrefour Forêts 2019, le colloque Sylviculture d'adaptation aux changements climatiques : des concepts à la réalité, organisé conjointement par la Direction de la recherche forestière du MFFP et le Centre canadien sur la fibre de bois de Ressources naturelles Canada, a rassemblé une centaine d'acteurs du milieu forestier incluant des ingénieurs forestiers, des biologistes, des techniciens forestiers, des propriétaires de boisés, des professeurs, des chercheurs et des étudiants. Des conférenciers de différentes régions du Québec et du Vermont ont partagé les connaissances actuelles en lien avec la sylviculture d'adaptation, afin d'amorcer la réflexion et le dialogue quant aux actions pouvant être entreprises dès maintenant pour aider les forêts à s'adapter aux changements anticipés. Le programme du col- loque $^{1}$ a été conçu de manière à couvrir les fondements théoriques de la sylviculture d'adaptation, pour ensuite présenter des réflexions ou des études de cas couvrant différents types d'écosystèmes, des outils innovants ou des initiatives de recherche. Finalement, le colloque a laissé une place aux praticiens qui ont pu décrire les défis et les occasions qu'offre la sylviculture d'adaptation dans leur pratique professionnelle.

L'ensemble des résumés et des présentations du colloque sont disponibles en ligne (Ministère des Forêts de la Faune et des Parcs 2019). Notre objectif, dans cet article, est de rassembler et de diffuser l'information qui a été partagée lors du colloque de manière à stimuler la réflexion et favoriser les échanges. Nous visons ainsi à soutenir la mise en œuvre d'une sylviculture qui aidera les forêts à s'adapter et à remplir le plein rôle qu'elles peuvent jouer dans la lutte aux changements climatiques (Lemprière et al. 2013).

\section{Évaluation intégrée de la vulnérabilité des forêts canadiennes aux changements climatiques}

Les espèces arborescentes varient dans leur capacité à tolérer, à migrer ou à s'adapter aux changements climatiques, ce qui aura des conséquences importantes sur la santé et la productivité des écosystèmes forestiers futurs, la composition des forêts et le régime des perturbations. Pourtant, les projections à large échelle de l'impact des changements climatiques sur les forêts prennent rarement en compte ces aspects de la vulnérabilité. Les chercheurs ont développé des indices basés sur les traits fonctionnels qui reflètent les différences dans la sensibilité et la capacité d'adaptation des espèces arborescentes aux principaux facteurs de stress climatiques (Fig. 1). Leurs travaux ont visé à déterminer des zones de vulnérabilité à la sécheresse et à l'échec de migration (incapacité pour une espèce de migrer assez rapidement pour suivre le déplacement de sa niche climatique) sur la composition et la productivité future des forêts canadiennes (Fig. 2). Ceci a été accompli en combinant des projections climatiques déterminant le degré d'exposition des forêts avec la sensibilité des espèces à la sécheresse et à l'échec de migration (Aubin et al. 2018) ${ }^{2}$. Cet exercice leur a permis de mettre en relief les différences régionales qui existent dans la vulnérabilité des forêts à ces deux éléments.

En plus du degré d'exposition et de la sensibilité des espèces, la vulnérabilité des forêts dépend également de la capacité des espèces à persister sur place dans de nouvelles conditions de croissance, c'est-à-dire de leur capacité d'adaptation (Spathelf et al. 2015). Les conférenciers ont développé un cadre conceptuel pour quantifier la capacité d'adaptation de 26 espèces arborescentes canadiennes selon quatre composantes d'adaptation : 1) le degré de plasticité phénotypique (la capacité d'un organisme à exprimer plusieurs caractéristiques selon différentes situations environnementales), 2) la diversité génétique, 3) les flux génétiques à l'intérieur des populations d'arbres et 4) ceux entre les populations.

Le développement d'outils spatiaux intégrant les informa-

\footnotetext{
${ }^{1}$ Le programme et les présentations sont disponibles ici : https://mffp.gouv.qc.ca/documents/forets/recherche/Actes Carrefour_Forets_2019.pdf

${ }^{2}$ Et voir : https://glfc.cfsnet.nfis.org/fcvul/?\&lang=fr
} 


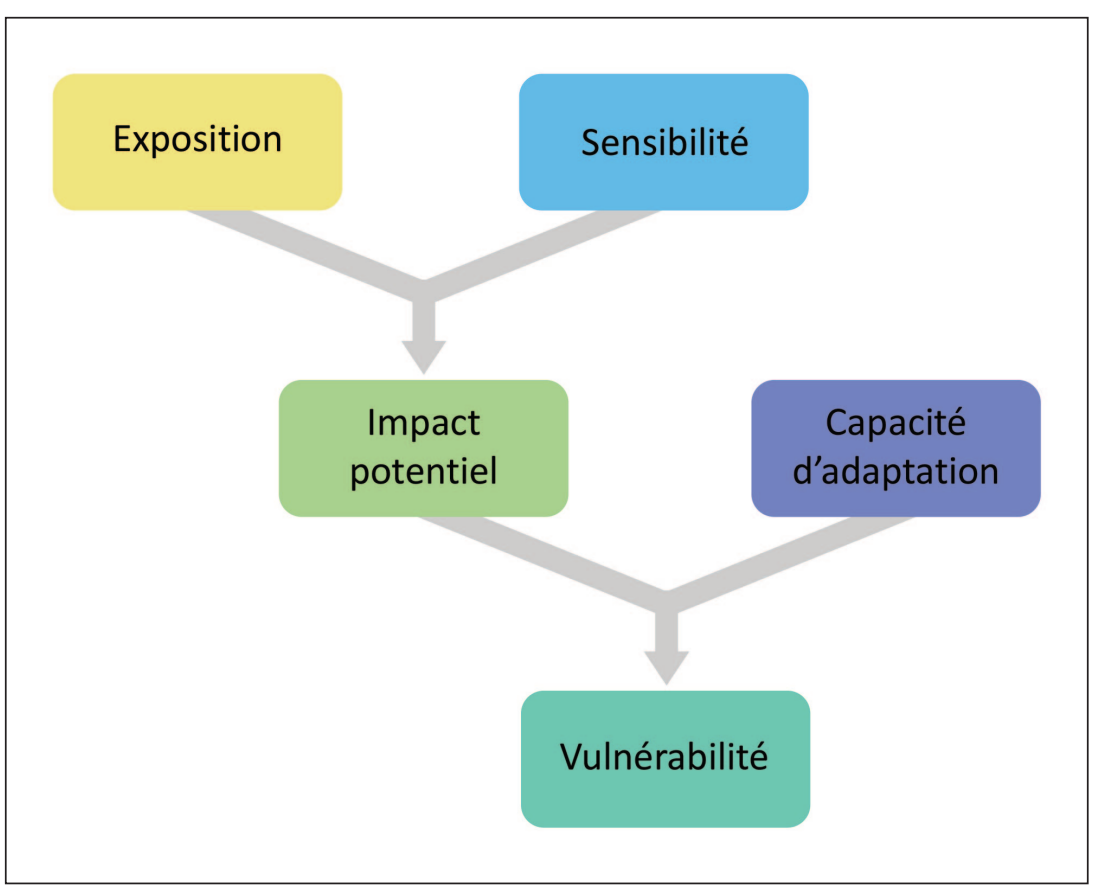

Fig. 1 La vulnérabilité des forêts aux changements climatiques englobe trois composantes principales : 1) le degré d'exposition des arbres à ces changements (p. ex : sécheresse, feu), 2) la sensibilité des espèces d'arbres à ces différents facteurs de stress et 3) la capacité des espèces à s'adapter aux nouvelles conditions créées. Ensemble, l'exposition et la sensibilité définissent l'impact potentiel qui, combiné à la capacité d'adaptation, détermine la vulnérabilité des forêts. Adapté de Glick et al. (2011).

tions relatives à la sensibilité et à la capacité de migration et d'adaptation des espèces est une étape critique dans l'élaboration de stratégies d'adaptation. Des travaux sont en cours en ce sens pour proposer un éventail d'options sylvicoles tenant compte de la vulnérabilité des écosystèmes et du principe de zonage forestier, de manière à promouvoir un aménagement forestier durable qui permet l'atteinte d'une multitude d'objectifs à l'échelle du territoire. Les conférenciers ont proposé plusieurs approches sylvicoles représentant un gradient d'intensité d'aménagement allant du maintien de réserves biologiques dans les refuges climatiques à l'utilisation de plantations à haut rendement dans un climat changeant. Cette dernière permettra de préserver l'approvisionnement en matière ligneuse tout en favorisant l'adaptation naturelle des espèces ainsi qu'une diversité de réponses des forêts aux changements attendus.

\section{Intégrer les dynamiques nouvelles et naturelles dans la conception de stratégies sylvicoles d'adaptation}

Maintenir la structure, les fonctions et les services fournis par les écosystèmes forestiers dans le contexte des changements globaux représente un défi important pour les aménagistes et les décideurs. Pour y faire face, une gamme d'approches d'adaptation a été proposée, allant de stratégies de résistance à des stratégies de transition. Leur efficacité opérationnelle à réduire les risques associés aux changements climatiques demeure toutefois incertaine. Malgré l'avancement des connaissances, certaines approches développées avant que l'aménagement pour l'adaptation ne soit au centre des préoccupations, comme l'aménagement écosystémique, sont devenues moins populaires. Par ailleurs, plusieurs exemples opérationnels de stratégies d'adaptation aux changements climatiques et aux effets des espèces envahissantes existent dans les écosystèmes nordiques et tempérés. De plus, l'intégration des connaissances acquises au sujet des dynamiques naturelles et des processus écologiques en contexte d'aménagement écosystémique est avantageuse. Même si, ultimement, les dynamiques nouvelles deviendront fort probablement dominantes, la compréhension des dynamiques naturelles demeure un point de départ logique pour guider le choix des méthodes de régénération, de conditions d'attributs structuraux, d'espèces et de traits fonctionnels à favoriser dans les stratégies d'adaptation.

Le choix des tiges à conserver lors de coupes de régénération est une considération importante des stratégies forestières d'adaptation (Ontl et al. 2018). Dans la plupart des cas, la sylviculture d'adaptation vise d'abord à promouvoir les essences adaptées aux conditions futures prédites par les projections climatiques. Cependant, l'émulation des régimes historiques de perturbations pour favoriser ces essences reste une approche valable. Par exemple, la majorité des essences qui sont décrites comme " adaptées pour le climat futur » dans les forêts décidues nordiques des États avoisinant les Grands Lacs et dans le NordEst des États-Unis sont classifiées comme intolérantes ou semi-tolérantes à l'ombre (Fig. 3; Handler et al. 2014; Janowiak et al. 2018). En revanche, les essences tolérantes à l'ombre, notamment l'érable à sucre (Acer saccharum

\section{L'adaptation aux changements climatiques : un concept aux multiples facettes.}

Le terme adaptation peut s'appliquer tant aux systèmes biologiques qu'aux systèmes humains (Gauthier et al. 2014) et peut référer à différentes réponses au changement. L'adaptation peut désigner la capacité inhérente d'un système biologique ou humain de s'ajuster et de s'adapter aux conséquences néfastes des changements climatiques. Cette capacité d'adaptation correspond à la troisième composante de la vulnérabilité. Le terme adaptation peut également qualifier les actions prises afin de limiter les impacts des changements climatiques sur les écosystèmes et les économies forestières. On parle alors de mesures, d'options ou encore de stratégies d'adaptation. Tout au long de cet article, le terme adaptation sera utilisé tour à tour dans l'un ou l'autre de ces contextes. 


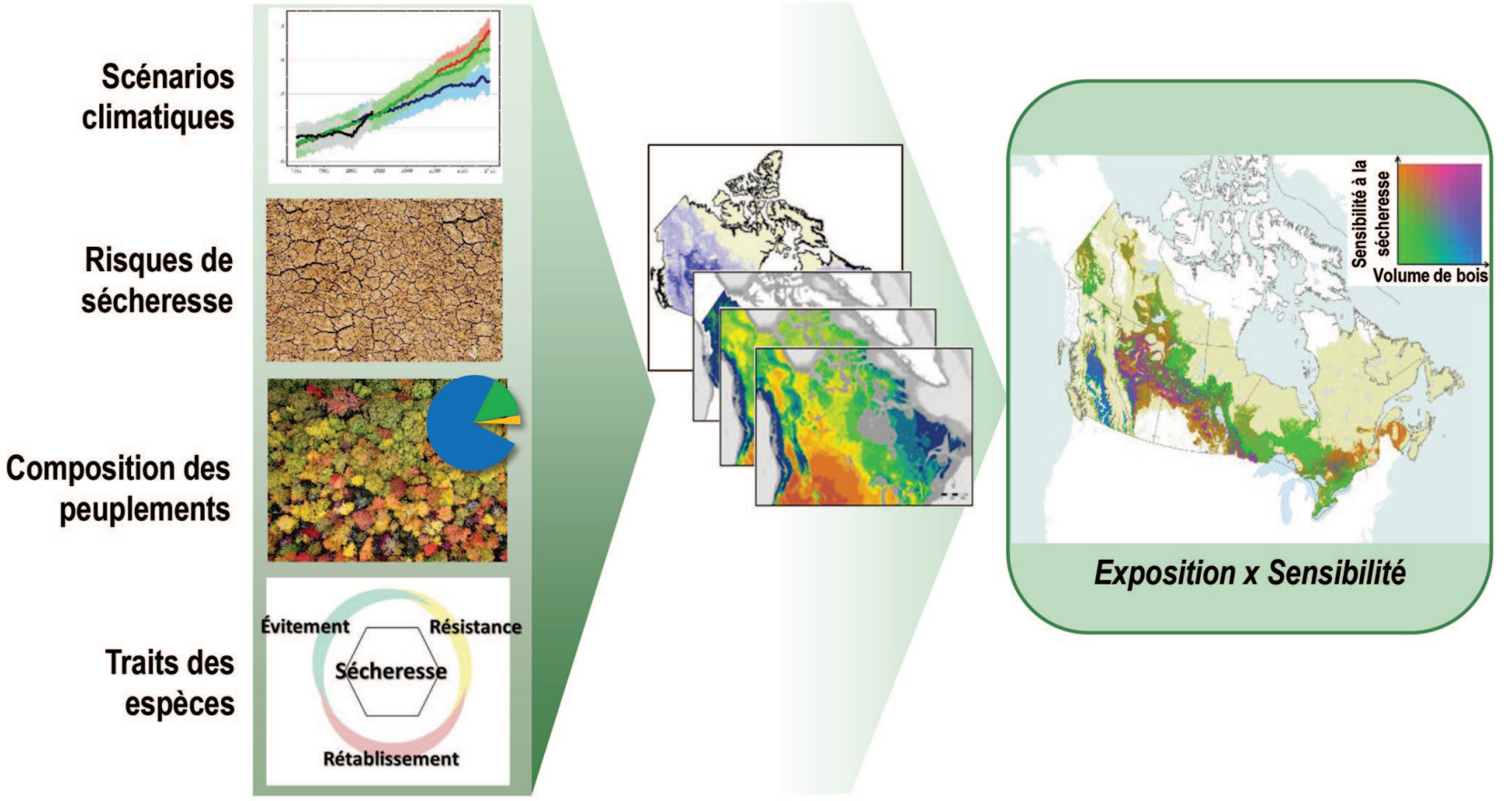

Fig. 2 Cadre conceptuel pour une évaluation intégrée de l'impact potentiel des changements climatiques sur les forêts canadiennes. Les prédictions biophysiques d'exposition sont intégrées aux connaissances écologiques sur la sensibilité des arbres (traits des espèces) et à la composition des peuplements afin de produire des cartes des endroits où la biomasse forestière est exposée et sensible aux facteurs de stress associés aux changements climatiques.

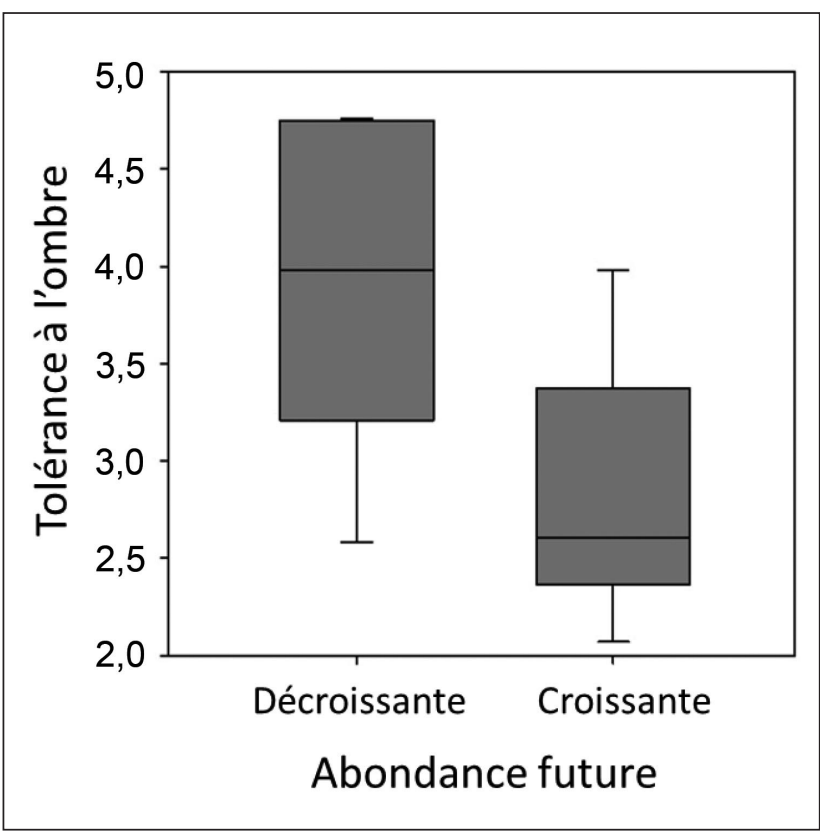

Fig. 3 Valeur de la tolérance à l'ombre des essences qui, selon les projections, vont vivre une décroissance et une croissance de leur abondance future au cours des 100 prochaines années dans le nord de la Nouvelle-Angleterre, dans le cadre d'un scénario d'émissions élevées fondé sur Prasad et al. (2007). Les valeurs de tolérance à l'ombre pour les essences vont de 1 (très intolérantes) à 5 (très tolérantes) et sont basées sur la classification de Niinemets et Valladares (2006).
Marsh.), sont favorisées par des systèmes sylvicoles basés sur la famille des coupes de jardinage, qui entraînent souvent l'exclusion d'essences moins tolérantes (Webster et Jensen 2007). Dans le passé, les perturbations intermédiaires comme les chablis causés par des tempêtes de vent se sont montrées essentielles à la création des conditions de recrutement nécessaires pour les essences intolérantes et semi-tolérantes à l'ombre de ces écosystèmes. L'émulation de ces patrons de dynamique naturelle par le biais de coupes progressives irrégulières offre l'occasion de recruter naturellement les essences adaptées à l'avenir, si elles sont présentes, ou de fournir des conditions adéquates pour des plantations d'enrichissement axées sur l'amélioration de la réponse fonctionnelle future (cf. Messier et al. 2019).

Forêt s'Adapter : un programme de recherche pour le développement d'une sylviculture de l'adaptation aux changements climatiques

Le programme de recherche appliquée Forêt s'Adapter, élaboré en partenariat avec les acteurs de la forêt privée de la Montérégie et de Bellechasse, a pour but d'acquérir des connaissances et de créer des outils pour la mise en place d'une sylviculture d'adaptation aux menaces des changements globaux. Une première phase du programme consiste à évaluer la vulnérabilité des écosystèmes forestiers la sensibilité des différentes espèces d'arbres aux menaces des changements globaux en cernant les facteurs d'exposition (Fig. 4a). Cela permet de distinguer les impacts selon leur probabilité de réalisation, leur spécificité (p.ex. : l'agrile du frêne qui s'at- 
a

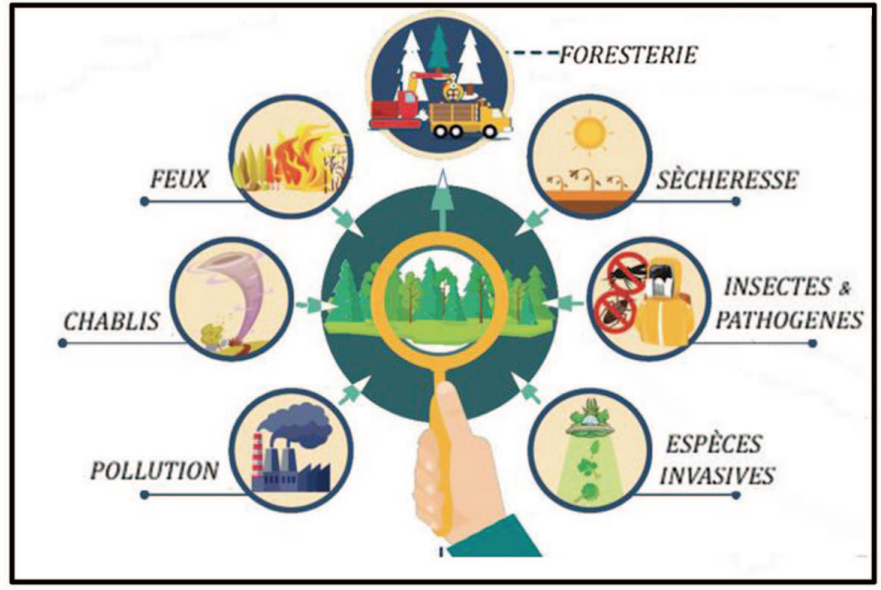

b

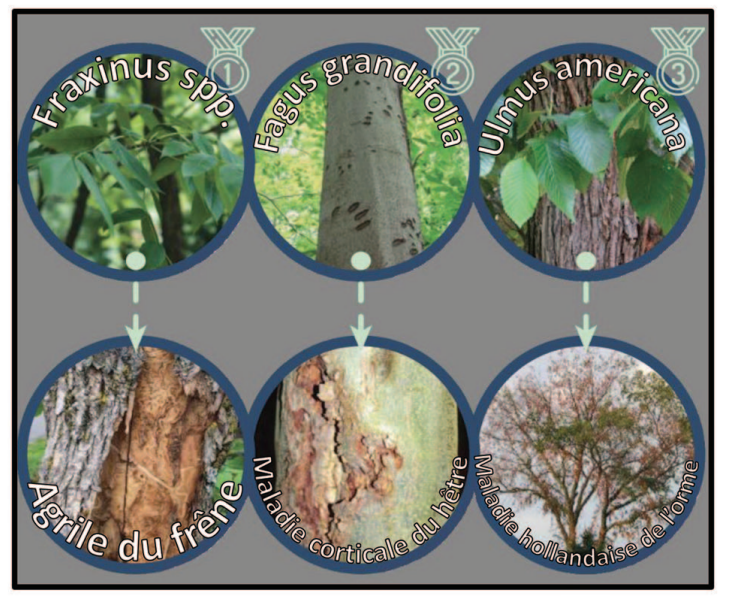

Fig. 4 Évaluation régionale (a) de l'exposition et (b) de la sensibilité des espèces aux menaces des changements globaux (images : Rita Sousa-Silva].

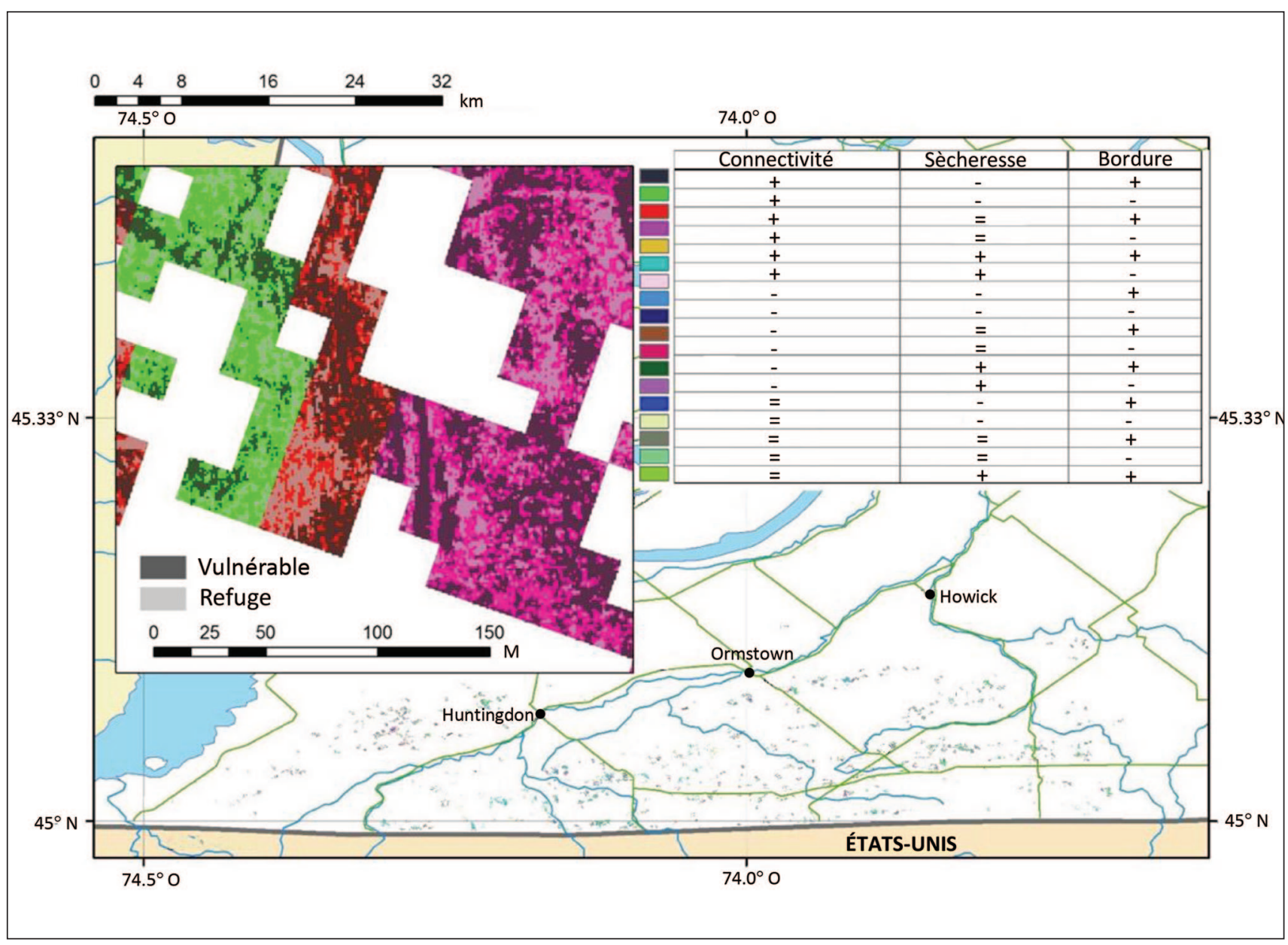

Fig. 5 Vulnérabilité des peuplements dans la région de la Vallée-du-Haut-Saint-Laurent (Québec). L'indice de vulnérabilité tient compte de la fragmentation, de l'effet de bordure et d'un complexe de variables physiographiques (microrelief, sol, etc.) pour estimer les risques associés au broutement du cerf de Virginie, aux invasions d'espèces exotiques et envahissantes ainsi qu'à la sécheresse. 


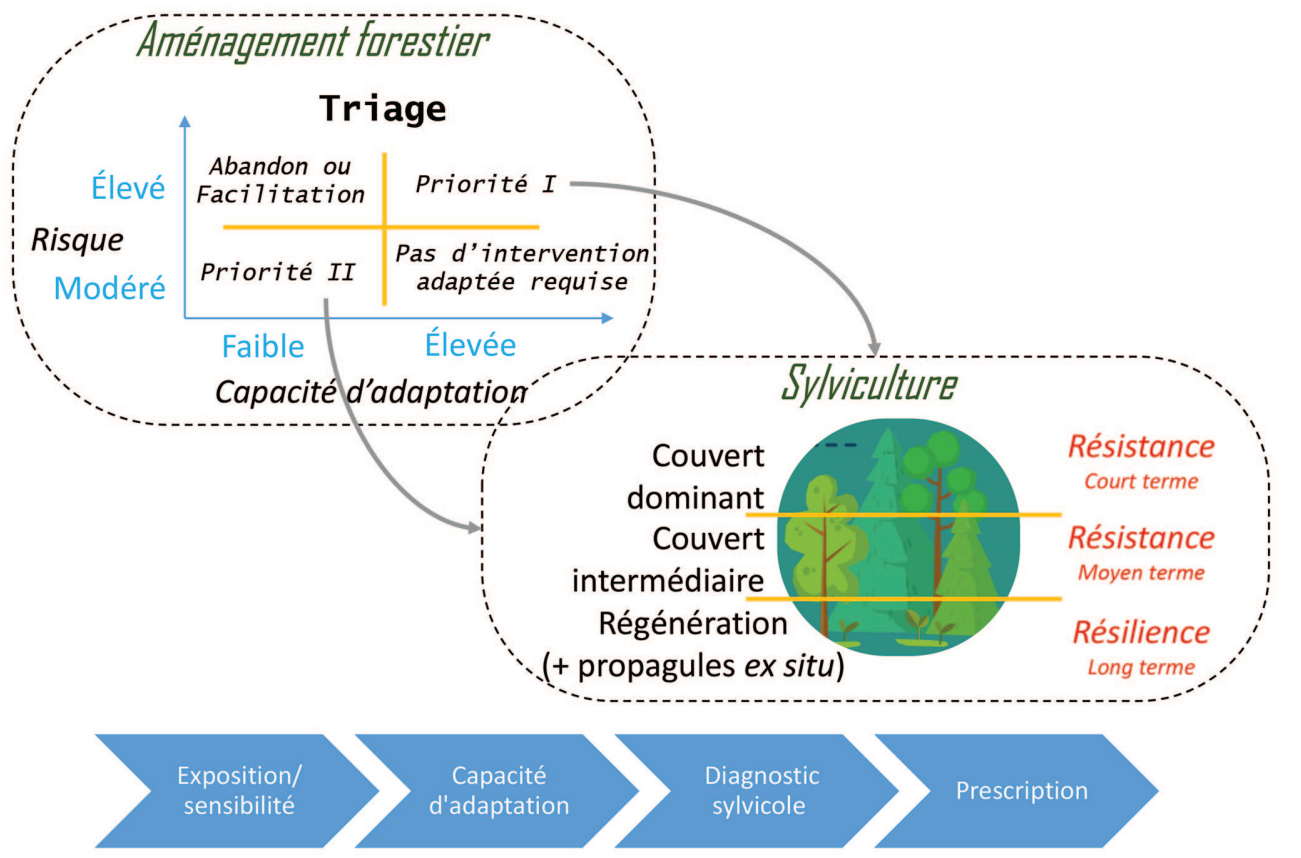

Fig. 6 La stratégie d'adaptation basée sur une grille de triage faisant intervenir le risque des menaces associées aux changements globaux et la capacité d'adaptation des peuplements permet d'optimiser les priorités d'intervention.

taque seulement au genre Fraxinus spp.; Fig. 4b) et leur gravité (perte de services écosystémiques) et de cartographier ceux-ci (Fig. 5). Sur la base de ces informations, une stratégie d'adaptation est développée et sert à trier les peuplements en quatre classes, de façon à optimiser les efforts d'adaptation (Fig. 6). Ce triage est basé sur l'importance du risque et la capacité d'adaptation des peuplements. Ainsi, pour les peuplements présentant un risque modéré et une capacité d'adaptation élevée, aucune mesure d'adaptation spécifique ne sera appliquée puisque l'on considère alors qu'ils pourront s'adapter d'eux-mêmes aux changements globaux les plus menaçants de la région; la sylviculture conventionnelle s'applique alors. De même, aucune mesure d'adaptation n'est recommandée pour les peuplements présentant un risque élevé et une faible capacité d'adaptation; l'investissement dans des traitements d'adaptation coûteux est jugé inutile, compte tenu du peu de matériel biologique en place pour affronter les menaces régionales actuelles et futures. Cette stratégie d'abandon mise sur la possibilité que les communautés forestières puissent se réorganiser d'elles-mêmes en de nouveaux assemblages mieux adaptés. Dans des forêts se trouvant dans cette classe d'adaptation, mais auxquelles on reconnaît une forte valeur socio-économique, une stratégie facilitant la transformation (sensu Millar et al. 2007) peut être mise en place afin d'accélérer la réorganisation d'un nouvel assemblage jugé mieux adapté. Les efforts d'adaptation sont accordés dans les deux autres classes : en premier lieu, les peuplements présentant un risque élevé et une capacité d'adaptation élevée et ensuite, ceux présentant un risque modéré et une capacité d'adaptation faible (Fig. 6). Pour ces deux groupes, le sylviculteur peut travailler en résistance à court terme avec le couvert supérieur, en résistance à moyen terme avec le couvert intermédiaire, ou en résilience à long terme avec la régénération potentielle (Fig. 6). Ce choix se fait lors du diagnostic sylvicole en fonction 1) des autres objectifs d'aménagement du propriétaire et 2) des traits fonctionnels des essences présentes dans le peuplement et de leur répartition dans ces trois strates de végétation (couvert dominant, couvert intermédiaire et régénération). Les traits fonctionnels choisis pour ce diagnostic sylvicole seront ceux conférant de la capacité d'adaptation aux menaces régionales des changements globaux définies à la phase 1 .

En collaboration avec l'Agence forestière des Bois-Francs ainsi que l'Agence de mise en valeur des forêts privées des Appalaches, cette approche a été rendue opérationnelle lors de la mise en place de deux dispositifs de sylviculture d'adaptation (dispositif SylvAdapt), situés au centre du Québec (15 sites) et en Bellechasse (12 sites). Deux stratégies d'adaptation ont alors été comparées soit : 1) l'approche du diagnostic sylvicole basée sur la capacité d'adaptation des espèces aux menaces régionales, telle que précédemment illustrée et 2 ) celle la police d'assurance de la diversité (compositionnelle, fonctionnelle et structurale). Dans chaque site, 4 parcelles de 0,25 ha ont été traitées selon 3 modalités : $20 \mathrm{~m}^{2} \cdot \mathrm{ha}^{-1}$ de surface terrière $(\mathrm{G})$ résiduelle pour le traitement de résistance court terme (témoin), $12 \mathrm{~m}^{2} \cdot \mathrm{ha}^{-1} \mathrm{de} \mathrm{G}$ résiduelle pour le traitement de résistance moyen terme et $6 \mathrm{~m}^{2} \cdot \mathrm{ha}^{-1}$ de $\mathrm{G}$ résiduelle pour le traitement de résilience (pour un total de 108 parcelles; Fig. 7a et Fig. 7b). Dans chaque parcelle, tous les arbres ont été cartographiés à l'aide du lidar mobile ter- 
a

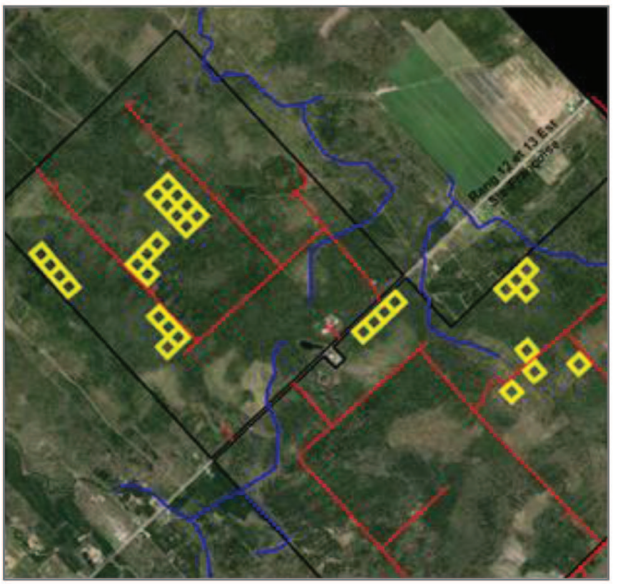

C

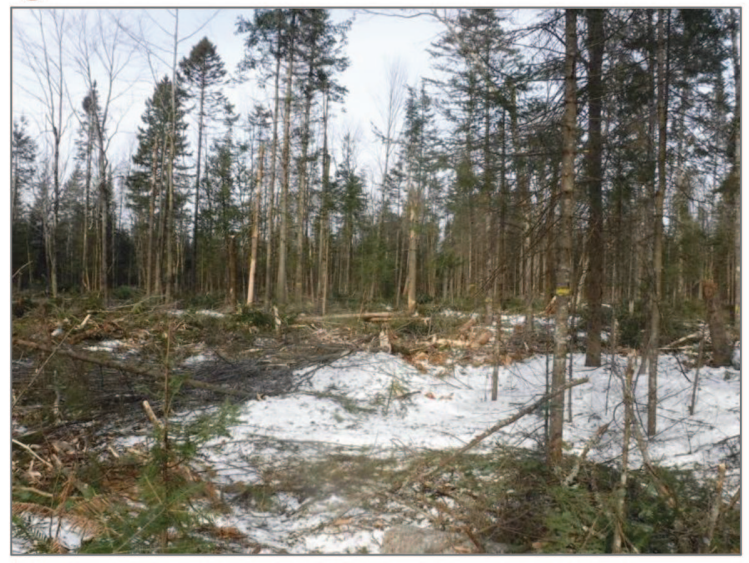

b
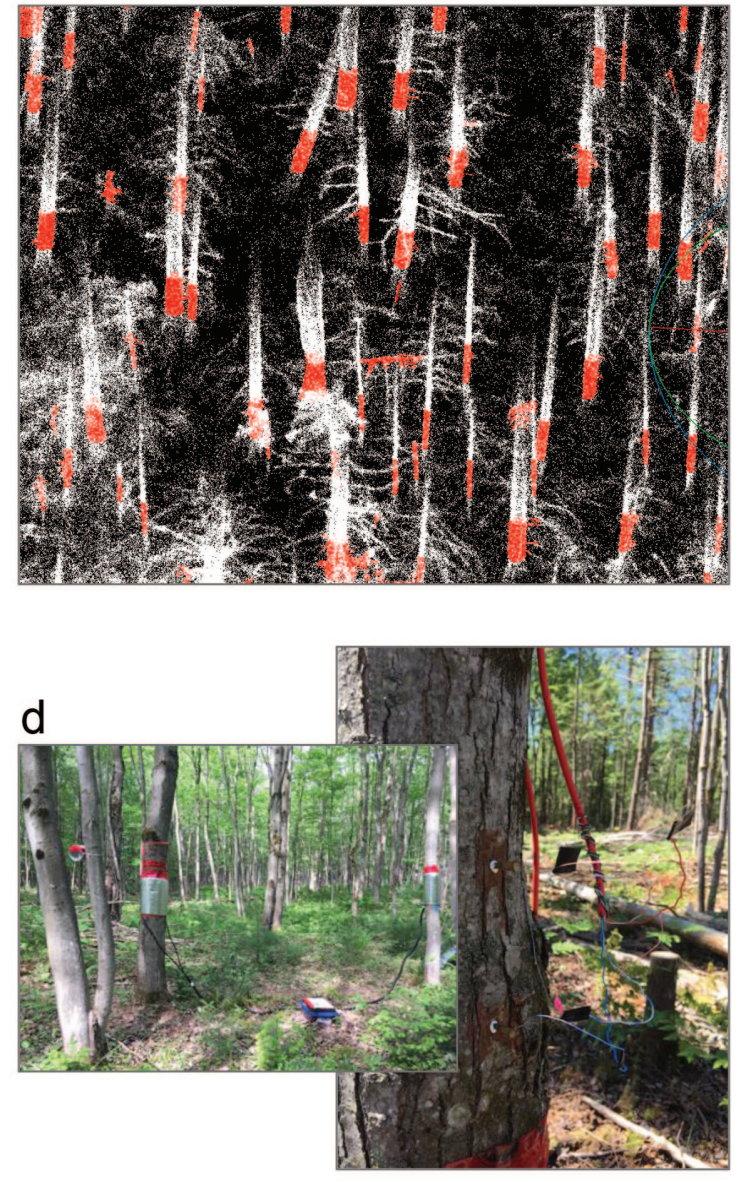

Fig. 7 Dispositif expérimental SylvAdapt de sylviculture d'adaptation. (a) Plan du dispositif à Sainte-Françoise; (b) image lidar d'une partie d'une parcelle avant traitement; (c) exemple de parcelle traitée en résistance à moyen terme avec la stratégie de la police d'assurance de la diversité; (d) érables rouges instrumentalisés avec une sonde de mesure de flux de sève (photos : Frédérik Doyon).

restre (env. 50000 arbres) avant et après traitement (Fig. 7c). Plusieurs projets de recherche gravitent autour de ce dispositif expérimental de sylviculture d'adaptation. Certains arbres sont munis d'instruments pour le suivi du flux de sève afin d'évaluer leur réponse aux stress du traitement et de la sécheresse ainsi le bilan hydrique du peuplement (Fig. 7d). De plus, des semis de 8 essences feuillues ont été plantés dans chaque parcelle : certaines localement abondantes (Acer rubrum L., Betula alleghaniensis Britt.), d'autres présentes, mais moins fréquentes (Acer saccharum Marsh., Prunus serotina Ehrh, Quercus rubra L.) et d'autres absentes régionalement (Quercus macrocapra Michx., Carya cordiformis (Wangenh.) K. Koch, Juglans nigra L.). Leur performance écophysiologique sera suivie dans les années qui viennent pour comparer l'effet des traitements de sylviculture d'adaptation.

\section{Options sylvicoles pour promouvoir une forêt mixte tempérée résiliente dans un contexte d'incertitude}

Le domaine bioclimatique de la sapinière à bouleau jaune (Betula alleghaniensis Britt.) constitue un écotone où coexistent des espèces de la forêt tempérée et de la forêt boréale.
Comme les changements climatiques surviendront à un rythme plus rapide que la capacité d'adaptation ou de migration des espèces, des pertes d'habitats sont anticipées chez les espèces boréales, en particulier chez les conifères (Iverson $e t$ al. 2008; Périé et al. 2014). Ces bouleversements risquent de se répercuter sur la composition, la structure et la productivité des écosystèmes (Gauthier et al. 2015). Des changements de composition sont appréhendés en particulier dans les forêts situées dans l'écotone tempéré-boréal (Reich et al. 2015). Les choix sylvicoles peuvent aider les forêts à s'adapter, en promouvant la diversité et la résilience ainsi qu'en facilitant la transition vers de nouveaux états (Millar et al. 2007). Dans les peuplements mûrs et surannés arrivant en fin de succession, on désire conserver la résistance et la résilience des forêts en misant sur la conservation d'arbres vigoureux, de semenciers d'essences en voie de raréfaction et de caractéristiques de vieilles forêts. Les procédés de régénération qui s'inspirent de la dynamique des perturbations naturelles, comme ceux par coupe de jardinage ou par coupe progressive irrégulière, pourraient contribuer à atteindre ce but (Raymond et Bédard 2017; Raymond et al. 2018). Les peuple- 


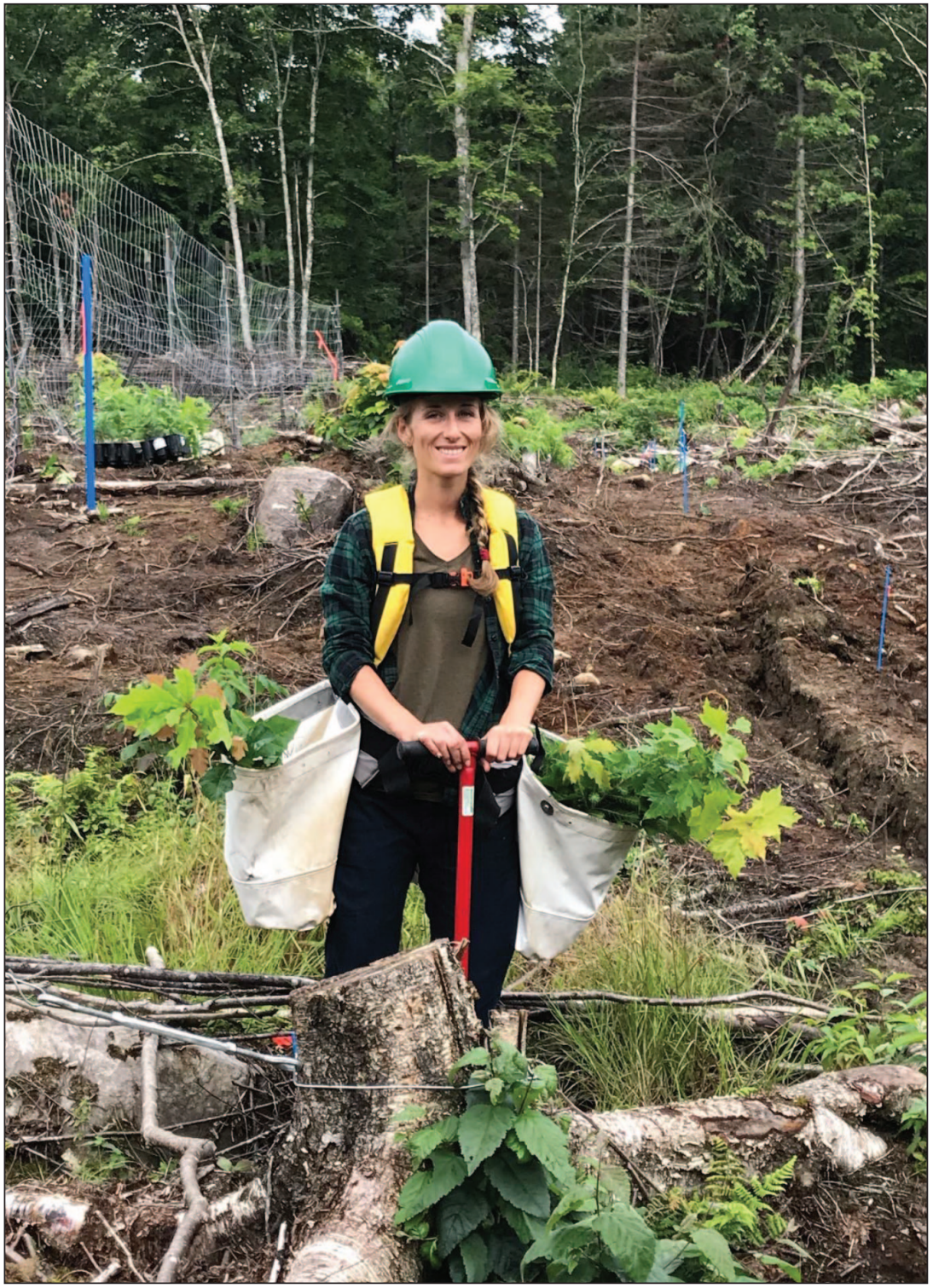

Fig. 8 Mise en place par la Direction de la recherche forestière du MFFP d'une expérience visant à tester des scénarios sylvicoles intégrant la migration assistée en forêt mixte (photo : Patricia Raymond).

ments d'âge intermédiaire comprennent ceux établis après une perturbation majeure d'origine naturelle (feu, épidémie d'insecte ou chablis) ou anthropique (coupe). La résistance et la résilience pourraient être améliorées par la promotion de la diversité des espèces et de la complexité structurale (Puettmann et Messier 2019). Le maintien de la composante résineuse et l'établissement de plus d'une cohorte de régénération dans le temps, à l'aide de procédés par coupes progressives (irrégulières ou régulières), pourraient aider à atteindre ces objectifs (Raymond et al. 2009; Prévost et DeBlois 2014). Comme les peuplements en phase de régénération subiront les effets des changements climatiques durant la plus grande partie de leur vie, il serait sage de planifier aussi des options de transition. Les traitements sylvicoles favorisant la diversité et l'adaptabilité pourraient aider à mitiger les effets des changements climatiques sur les jeunes peuplements. La sélection des arbres lors de traitements sylvicoles contribuera à réduire la vulnérabilité de ces peuplements dans le futur. L'utilisation d'éclaircies précommerciales mixtes et de plantations mixtes pourrait s'avérer judicieuse pour promouvoir la diversité fonctionnelle et maximiser l'adaptabilité. On favorisera le choix d'arbres vigoureux, d'essences tolérantes à la sécheresse ou de provenances compatibles avec le climat futur. Enfin, la migration assistée d'essences et de provenances fera vraisemblablement partie des stratégies d'adaptation aux changements climatiques (Ste-Marie et al. 2011). La recherche se poursuit pour en apprendre davantage sur les conditions de succès de ces plantations, en vue de les intégrer à la planification forestière (Fig. 8).

\section{Enjeux et possibilités de la sylvicul- ture d'adaptation en forêt boréale}

La forêt boréale est un biome particulièrement sensible aux changements climatiques (Gauthier et al. 2015). Un climat plus chaud et sec entraînera ultimement une diminution de la productivité et une augmentation de la mortalité (D'Orangeville et al. 2018). Ces changements se produiront plus rapidement que la vitesse d'adaptation des espèces; ils pourraient ainsi mener à une simplification des écosystèmes (Brandt et al. 2013). Les changements climatiques influenceront aussi le régime des perturbations et pourraient compromettre la capacité des forêts boréales à se régénérer, ce qui soulève de nombreux enjeux (Fig. 9). Particulièrement, les stress physiologiques associés à des modifications des régimes de précipitations sont susceptibles de causer une mortalité accrue de la régénération forestière.

La sylviculture d'adaptation pourrait permettre de mitiger les effets des changements climatiques en forêt boréale en favorisant la résistance, la résilience ou encore la transition des écosystèmes boréaux, particulièrement au stade de la régénération. L'épinette noire (Picea mariana (Mill.) B.S.P.) sera parmi les essences commerciales les plus affectées par la hausse des températures et les déficits en eau (Boucher et al. 2020). Étant donné l'importante place que cette essence occupe dans les couverts forestiers boréaux, de faibles taux de survie pourraient occasionner une hausse des accidents de régénération. Des études en cours permettront, notamment, de mieux comprendre sa réponse aux stress hydriques, particulièrement à l'égard des problèmes de cavitation (la formation de bulles d'air dans la colonne d'eau) (Fig. 10). Des pratiques sylvicoles innovantes, comme la plantation d'essences compagnes ( "nurse plant species») en plus de la préparation mécanique du sol, ont quant à elles démontré leur potentiel pour stimuler la croissance des arbres mis en terre dans les milieux boréaux où les conditions de croissance sont limitantes (Thiffault et Hébert 2017; Urli et al. 2020). La planta- 


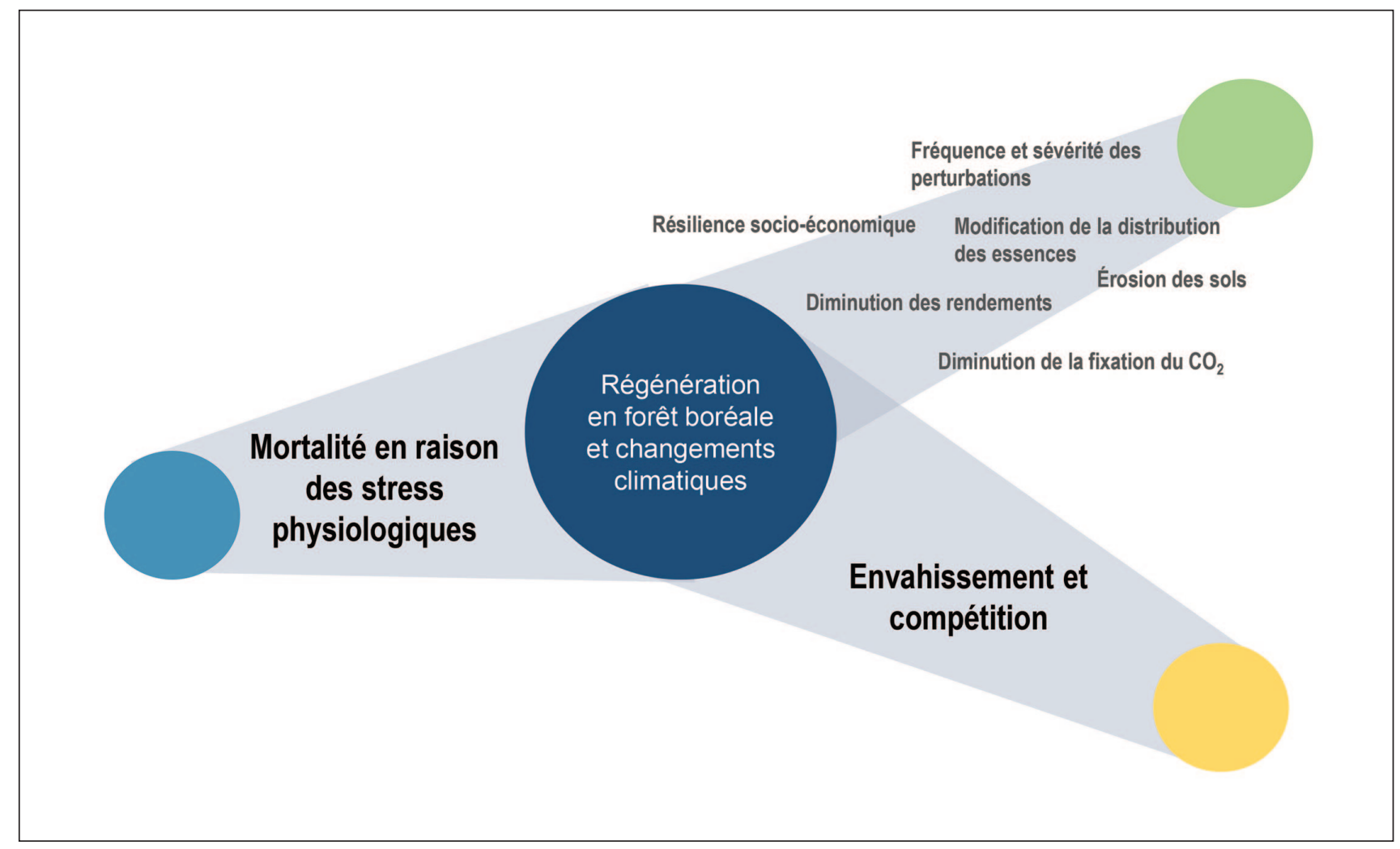

Fig. 9 Certains enjeux associés aux les changements climatiques au regard de la régénération et du maintien de la productivité en forêt boréale.

tion mixte avec des essences moins sensibles aux extrêmes de sécheresse pourrait limiter les pertes de productivité. Les changements climatiques ont par ailleurs le potentiel de modifier la phénologie et la répartition des espèces concurrentes (Fridley 2012). Les pratiques de gestion de la végétation devront donc être adaptées, notamment pour tenir compte de l'expansion vers le nord d'espèces exotiques envahissantes comme le nerprun (Frangula alnus Mill.) (Labonté et al. 2020).

Foresterie d'adaptation en zone de transition : le cas de la forêt d'enseignement et de recherche du Lac Duparquet (FERLD)

Importante pour le Québec sur les plans économique et écologique, la forêt boréale mixte constitue une zone de transition entre la forêt tempérée, dominées par les feuillus, et la forêt boréale résineuse. La dynamique naturelle de cette zone forestière est principalement contrôlée par les feux de forêt et les épidémies d'insectes (c'est-à-dire la tordeuse des bourgeons de l'épinette et la livrée des forêts; Morin et al. 1993; Bergeron 2000). En Abitibi, les sites mixtes de cette zone forestière sont typiquement dominés par le peuplier faux-tremble (Populus tremuloides Michx.) ou le pin gris (Pinus banksiana Lamb.) en début de succession, puis par le sapin (Abies balsamea (L.) Mill.) et le thuya (Thuya occidentalis L.) en fin de succession. Par ailleurs, certaines espèces typiques des forêts décidues, par exemple l'érable à sucre, le bouleau jaune, le pin blanc (Pinus strobus L.) et le pin rouge (Pinus resinosa Ait.), y atteignent la limite nordique de leur aire de répartition, alors que d'autres pourraient s'y établir, par exemple le tilleul d'Amérique (Tilia americana L.) et le chêne rouge. Dans ce contexte, toute modification au régime des perturbations pourrait favoriser la dispersion et l'établissement de certaines de ces essences marginales, particulièrement celles adaptées à un régime de perturbations qui comprend différents degrés de sévérité (Frelich 2002). Puisque les changements climatiques anticipés sont susceptibles de modifier le régime des perturbations en forêt boréale mixte, ces modifications pourraient aussi entraîner un changement dans la composition des peuplements et altérer davantage le régime des perturbations pour permettre l'établissement durable d'espèces marginales (Flannigan et al. 2000). Afin de tenir compte de ces modifications potentielles de la composition des forêts, le développement de nouvelles approches sylvicoles (par exemple, la coupe progressive irrégulière, des traitements d'éclaircies ou l'amendement des sols) ou la sélection de génotypes pourraient être nécessaires. La FERLD constitue un laboratoire où ces approches pourraient être testées.

\section{Contributions de la génétique forestière au reboise- ment : passé, présent et futur}

La génétique forestière permet de décrypter les différences entre individus d'une même espèce par rapport à la variabilité génétique naturelle. Bien que méconnues du public, ces connaissances sont maintenant intégrées dans l'amélioration des arbres et la production de semences et de plants au Québec (Perron et al. 2020). Dès les années 1950, des tests de provenances ont été établis pour plusieurs espèces indigènes. Ils ont permis de délimiter des zones d'amélioration et de choisir 


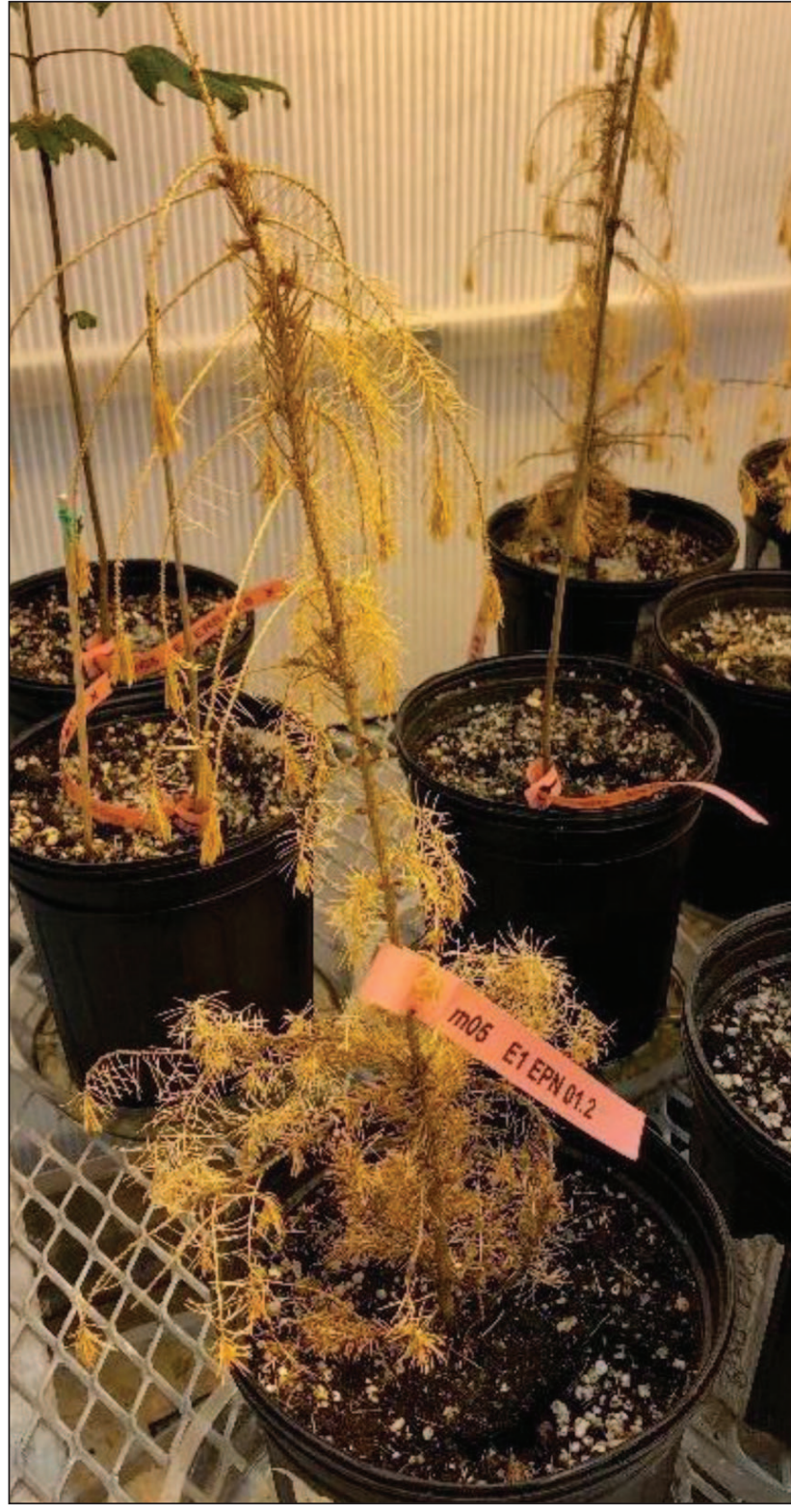

Fig. 10 Plants d'épinette noire dans une expérience en serres à I'Université Laval. L'objectif de cette étude, à laquelle collaborent Morgane Urli (Université Laval), Alison Munson (Université Laval), Marie Coyea (Université Laval), Catherine Périé (Ministère des Forêts, de la Faune et des Parcs du Québec] et Nelson Thiffault (Ressources naturelles Canada), est de comparer les réponses physiologiques et la vulnérabilité à la sécheresse de plants de cinq essences tempérées nordiques et boréales soumises à différentes conditions climatiques (photo : Nelson Thiffault).

les provenances les plus performantes et les mieux adaptées aux conditions des sites de reboisement. Par ailleurs, les nombreux tests de descendances établis ont permis d'estimer la valeur d'amélioration de centaines de milliers de parents candidats à la sélection en fonction de la productivité et la qualité du bois. Aujourd'hui, au Québec, $85 \%$ des plants utilisés pour le reboisement sont issus des programmes d'amélioration génétique. Plus récemment, les résultats des tests de provenances ont servi à développer des modèles de transfert ( $\mathrm{p}$. ex. Rainville et al. 2014) en vue d'optimiser l'adaptation locale aux changements climatiques selon des territoires d'utilisation qui sont dynamiques, c'est-à-dire qui tiennent compte $\mathrm{du}$ fait que les populations pourraient devenir mésadaptées à leur climat local (Fig. 11). La sélection génomique est progressivement intégrée chez les épinettes, par exemple chez l'épinette de Norvège (Picea abies (L.) Karst.) (Lenz et al. 2020). La génomique permet de confirmer que l'adaptation au climat provient d'une multitude de gènes à faibles effets et que de multiples régions du génome sont impliquées dans les processus de débourrement, de croissance et d'aoûtement (Pavy et al. 2017). L'analyse dendrochronologique de nombreuses descendances et provenances d'épinette blanche a aussi engendré la découverte d'une variation génétique importante pour la résistance au stress hydrique (Depardieu et al. 2020). Bientôt, l'amélioration génétique intégrera progressivement la sélection multicritère assistée par la génomique. Ainsi, des caractères difficiles à mesurer et important pour l'adaptation seront davantage pris en considération (Perron et al. 2020). On envisage aussi de recourir au testage multisite sur de plus grands gradients climatiques pour adapter les modèles de transfert et mieux détecter la variation génétique liée à l'adaptation, jusqu'ici difficile à cerner. En conséquence, ceci permettra de maintenir la productivité et la résilience des arbres en plantation. Conjointement, ces approches accéléreront l'acquisition des connaissances sur les caractères liés à l'adaptation, de manière à soutenir encore le reboisement dans le contexte des changements climatiques.

\section{Les défis de la mise en œuvre de la migration assistée proposée comme mesure d'adaptation aux changements climatiques}

Pour le reboisement au Québec, chaque source de semences (provenance) est associée à un territoire d'utilisation précis où sont déployés les plants. Le principe sous-jacent est que la source locale est la mieux adaptée. Or, les changements climatiques remettent en question ce postulat. La migration assistée consiste à déplacer des espèces ou des populations dans des habitats où les conditions climatiques à venir pourraient leur être plus favorables (Fig. 12). Au Québec, ce déplacement se fait généralement du sud vers le nord. La migration assistée représente ainsi une option sylvicole pour maintenir la productivité et la résilience des arbres en plantation. Néanmoins, les plants mis en terre aujourd'hui doivent survivre sous le climat actuel avant de pouvoir performer sous un climat futur; la mise en ouvre de la migration assistée doit donc aussi tenir compte du risque de mésadaptation au stade juvénile, qui augmente avec la distance de déplacement.

La migration assistée actuellement envisagée au Québec consiste à déplacer des populations au sein de l'aire de répartition de l'espèce afin de minimiser la mésadaptation génétique dans le futur, sans compromettre leur survie les premières années suivant la mise en terre (Ste-Marie et al. 2011). Sa mise en ouvre pour l'épinette blanche est en cours, et se traduit par l'actualisation des territoires d'utilisation modélisés pour un climat moyen en 2050.

Dans le cas où la migration assistée serait utilisée pour introduire des espèces dans des régions où elles ne sont pas encore présentes aujourd'hui (" assisted range expansion »; Ste-Marie et al. 2011), mais où les conditions climatiques anticipées leur deviendront favorables dans le futur, plusieurs 


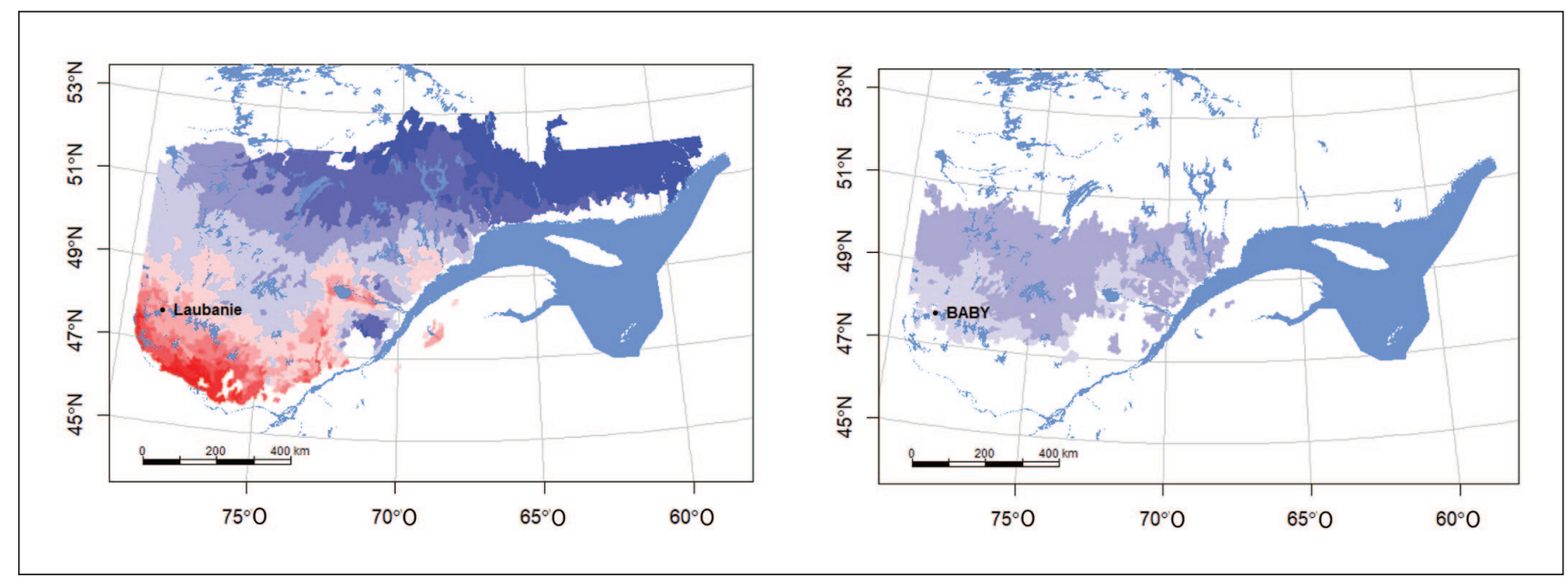

Fig. 11 Exemples de simulations de différences en volume marchand en fonction des variables climatiques de territoires d'utilisation dynamique pour l'épinette noire (à gauche) et l'épinette blanche là droite). L'échelle de couleurs va du bleu (gain en volume marchand] au rouge (perte en volume marchand). Modèle de transfert : Rainville et al. (2014); simulations : M.-C. Lambert, MFFP.

autres questions restent encore sans réponse. Les limites biophysiques, notamment la fertilité des sols, sont à considérer lors de la planification de tels déplacements. La migration assistée, outil prometteur d'adaptation des plantations aux changements climatiques, devra toutefois être déployée avec prudence et en intégrant les nouvelles connaissances au fur et à mesure qu'elles deviennent disponibles (Aubin et al. 2011).

\section{Les défis de la mise en œuvre des stratégies d'adap- tation : point vue des aménagistes}

La résilience des forêts décidues et mixtes est au cœur des préoccupations actuelles à l'égard des changements annoncés. La connectivité des massifs forestiers et la complexité des peuplements, tant par leur structure que par leur composition, sont les éléments clés susceptibles d'aider les forêts à s'adapter aux changements présents et futurs. D'autres éléments comme la modification des régimes de précipitations et de feux, ou encore les perturbations humaines, doivent s'ajouter à ces considérations. En tant que praticiens, les forestiers ne sont-ils pas déjà dépassés par ce phénomène? Une réflexion s'impose sur leurs réelles capacités d'intervention.

L'effervescence créée en 2013 lors de la mise en œuvre du nouveau régime forestier a mis à l'épreuve la capacité d'adaptation des professionnels de la forêt. La multitude des enjeux à concilier, la nécessité de considérer les contextes économique, social, environnemental et organisationnel pour se positionner et l'acquisition accélérée des connaissances ont tôt fait d'en étourdir plus d'un. Les défis qui se sont présentés alors permettent d'entrevoir l'ampleur de ceux découlant de la nécessité de s'adapter au contexte des changements climatiques. Les acteurs de la forêt seront-ils en mesure de déployer leur capacité d'adaptation pour y donner suite? Les conditions gagnantes seront-elles au rendez-vous?

\section{Synthèse : perceptions, risques et possibilités liés aux changements climatiques}

Il est évident que les perceptions relatives aux risques et aux possibilités liées aux changements climatiques pèsent lourd dans le développement de politiques et de programmes visant à favoriser l'adaptation de la forêt et à gérer ces changements (Williamson et al. 2005). Cela touche à la fois le pouvoir politique, les gestionnaires, les scientifiques et la société en général. Pour clore le colloque, en guise de synthèse, les participants ont pris part à un exercice d'introspection pour dresser le portrait de leurs perceptions quant aux risques et aux possibilités pour le secteur forestier du Québec d'ici le prochain siècle. De manière interactive, une série de questions ouvertes et fermées ont été posées à l'auditoire, pour dresser la liste et estimer l'ampleur des risques et des possibilités perçus, et énumérer les solutions potentielles. L'application Web Mentimeter ${ }^{3}$ a été utilisée pour saisir les réponses des participants par le biais de leur téléphone intelligent, et discuter «à chaud » des résultats.

Quarante-quatre personnes ont participé au sondage. La plupart des répondants étaient issus du secteur gouvernemental (54\%) ou académique (35\%), en enseignement et recherche $(57 \%)$ et en planification ( $29 \%)$. Ces professionnels œuvrent en forêt boréale ( $25 \%)$, mixte (41 \%) et décidue (34\%).

À partir de la liste proposée (Fig. 13a), les participants ont classé la sécheresse en tête des risques et enjeux appréhendés au regard des changements climatiques; sont venus ensuite la capacité de migration limitée des arbres et le problème des espèces envahissantes. La volonté politique, l'acceptabilité sociale et la mise en œuvre de stratégies d'adaptation figurent parmi les enjeux ajoutés à la liste par les participants, tout comme les changements de conditions opérationnelles en saison hivernale et la capacité technologique d'affronter les changements. Le risque associé est jugé très élevé par la majorité des participants, avec une probabilité jugée forte et un impact jugé important (Fig. 13b).

À tout changement est associé des risques, mais aussi des possibilités. Le climat étant l'un des principaux facteurs limitants de la productivité forestière, on se peut se questionner, par exemple, sur les possibles avantages d'un climat différent sur la croissance des arbres. Parmi les possibilités perçues par les participants, notons celle de l'établissement et de la sylviculture de nouvelles espèces, associés à une possible augmen-

\footnotetext{
${ }^{3}$ www.mentimeter.com
} 


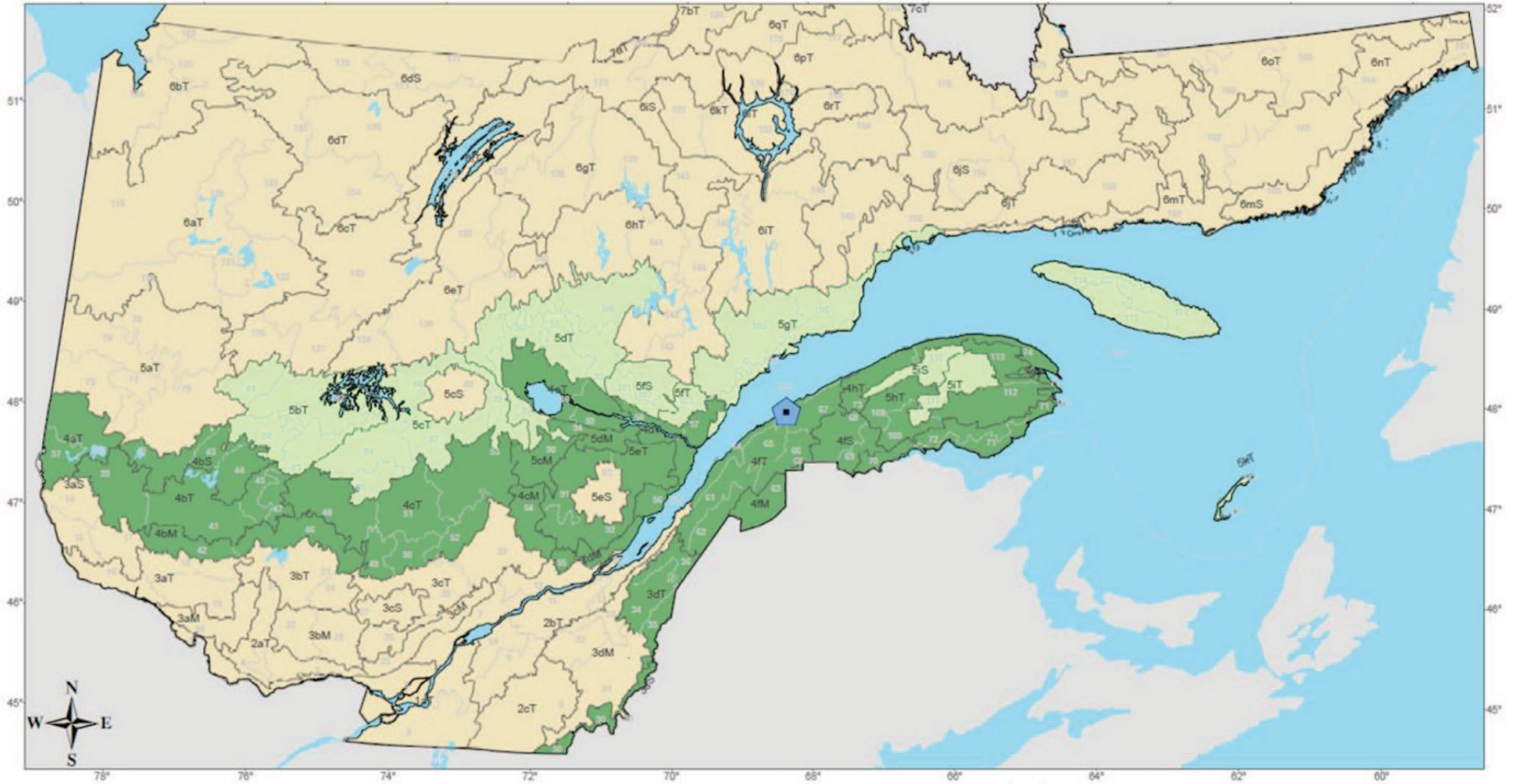

Fig. 12 En utilisant des mesures réalisées dans neuf sites de plantations tests réparties le long de trois transects sud-nord représentant un gradient climatique de $5,8^{\circ} \mathrm{C}$ à l'échelle du Québec (Lamhamedi et al. 2017), il a été possible d'actualiser le territoire d'utilisation du verger à graines de 2e génération d'épinette blanche de Sainte-Luce (vert foncé], en l'étendant vers le nord et vers l'est (vert pâle) (Benomar et al. 2018).

tation de la biodiversité. Ceci pourrait mener au développement de nouveaux marchés de produits, notamment dans l'économie verte émergente. La réponse de la forêt aux changements climatiques permettra aussi, peut-être, d'augmenter la contribution des forêts à la gestion des gaz à effet de serre et d'améliorer le positionnement du secteur forestier dans l'opinion publique. Plusieurs participants ont souligné que c'était là l'occasion de réviser et d'améliorer les pratiques de gestion forestière. Le niveau perçu d'opportunité, combinant la probabilité et l'importance de l'impact, varie beaucoup selon les participants, avec une tendance centrale en faveur d'un niveau d'opportunité élevée (Fig. 13c).

Plusieurs présentations du colloque ont proposé des mesures d'adaptation pour l'aménagement forestier. Les participants ont été invités à coter la pertinence des éléments d'une liste de solutions évoquées (Fig. 13d). La régénération et la gestion de peuplements mélangés, notamment par des plantations d'enrichissement en migration assistée, figurent en tête. Parmi les solutions supplémentaires proposées par les participants, plusieurs ont mentionné la réduction des périodes de révolution pour réduire l'exposition au risque dans le temps et l'adaptation progressive de la composition des peuplements au climat, en faisait appel aux essences à croissance rapide, à la fertilisation et à l'irrigation. L'implication de l'expérience et du savoir autochtones et l'allongement des révolutions pour augmenter la captation de $\mathrm{CO}_{2}$ ont aussi été mentionnés.

L'exercice a permis de dresser un portrait des perceptions et des priorités des chercheurs et professionnels forestiers présents au colloque. Les éléments présentés recoupent les observations d'études plus rigoureuses menées récemment
(Capstick et al. 2015; Morin et al. 2015; Bissonnette et al. 2017; Ameztegui et al. 2018).

\section{Conclusion}

Le colloque Sylviculture d'adaptation aux changements climatiques: des concepts à la réalité a permis de comprendre l'effet des changements globaux anticipés sur les processus physiologiques et écologiques qui déterminent la dynamique forestière, de même que leurs effets anticipés sur la production de services écosystémiques. Le colloque a contribué directement à l'effort de transfert des connaissances auprès des sylviculteurs, notamment en présentant des pratiques sylvicoles et des conditions de mise en ouvre favorisant l'adaptation des forêts aux changements climatiques. Les conférences de la journée ont abordé la question de la sylviculture d'adaptation, d'abord en expliquant des concepts théoriques, puis en proposant des solutions concrètes bien ancrées dans la réalité. Elles ont renseigné sur les différentes sensibilités et capacités d'adaptation des espèces d'arbres, information importante pour mieux comprendre la vulnérabilité aux changements climatiques des forêts québécoises. Les participants ont ensuite réfléchi à la place de l'aménagement écosystémique dans les stratégies d'adaptation, notamment sur le principe de l'éventail d'approches d'adaptation (résistance, résilience et transition) à l'aide de scénarios d'adaptation expérimentés aux États-Unis dans les peuplements décidus nordiques du New Hampshire. Il a ensuite été question des plus récentes connaissances issues du programme de recherche Forêt S'Adapter, dans les forêts décidues du sudouest du Québec, lequel programme s'articule autour des concepts de résistance, de résilience et de transition. Le col- 


\section{a}

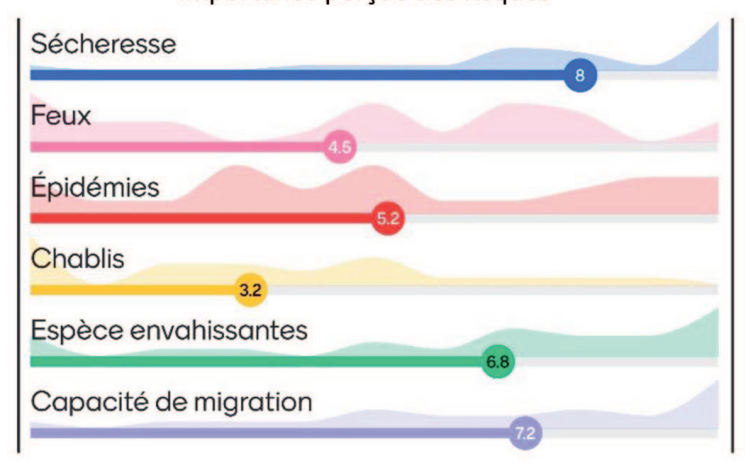

C

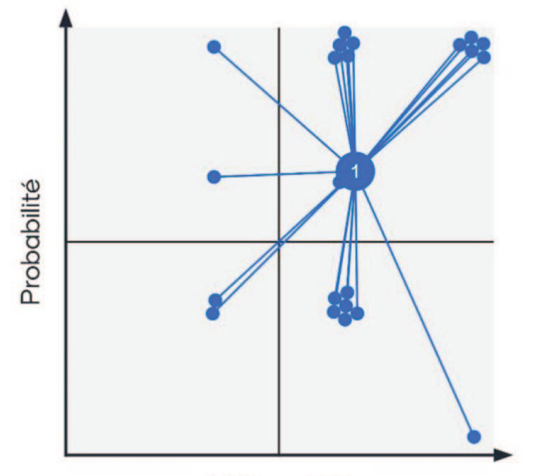

Effet positif

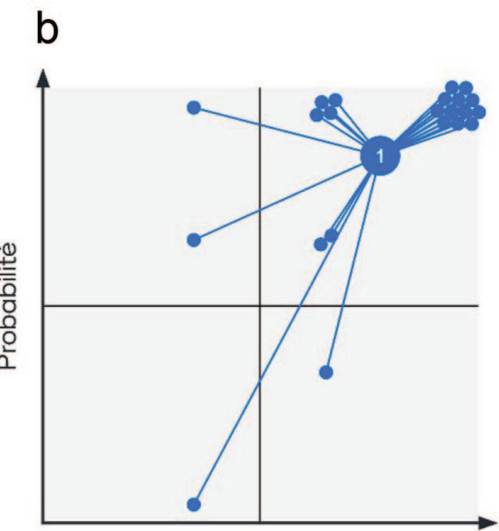

Effet négatif

Pertinence perçue des solutions

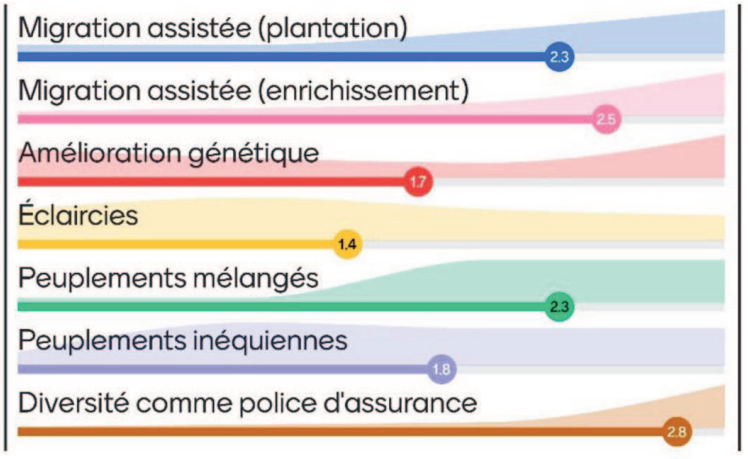

Fig. 13 Synthèse de la perception qu'ont les participants du colloque des risques et possibilités associés par les changements climatiques pour le secteur forestier du Québec au cours du prochain siècle. Le graphique (a) présente la distribution de fréquence de l'importance (de 1 = faible à 10 = forte) attribuée à une liste d'enjeux. Le graphique (b) est la matrice de risques, pour laquelle les participants ont coté à la fois l'impact et la probabilité des risques énumérés. Le graphique (c) présente le niveau d'opportunité perçu des différentes possibilités, à la fois quant à leur impact et à leur probabilité. Le graphique (d) illustre le niveau de pertinence perçu de quelques solutions présentées lors du colloque pour adapter l'aménagement forestier aux changements climatiques (selon une échelle de 1 = faible à 3 = élevé . Les graphiques (b) et (c) montrent à la fois la tendance centrale et la dispersion des scores.

loque a ensuite abordé des sujets un peu plus pratiques avec une série de présentations portant sur des exemples de traitements sylvicoles pouvant être appliqués dans un contexte d'adaptation. Il a été question d'options d'adaptation en fonction des stades de développement des peuplements pour la forêt mixte tempérée, d'exemples issus de la forêt boréale et d'options pour la forêt mixte boréale, en utilisant l'exemple de la FERLD. Ces présentations ont illustré comment les concepts de sylviculture d'adaptation visant la résistance, la résilience ou la transition des peuplements s'appliquent à différentes réalités bioclimatiques du Québec.

En après-midi, une séance de présentations éclair d'affiches a abordé des questions très variées en lien avec la sylviculture d'adaptation, allant de la physiologie des plants et de la résistance au broutement jusqu'au diagnostic et aux prescriptions sylvicoles ${ }^{4}$. Le colloque a ensuite exploré comment les progrès en amélioration génétique peuvent contribuer à la mise en ouvre de stratégies de transition, par exemple la

${ }^{4}$ Les présentations éclair sont disponibles ici :

https://mffp.gouv.qc.ca/documents/forets/recherche/Actes_Carrefour_Forets_2019.pdf migration assistée. Il a ensuite exploré les défis que représente la mise en œuvre de telles stratégies, notamment quant au choix des essences et des provenances pour la production de plants compatibles avec le climat futur. Par la suite, deux ingénieurs forestiers d'expérience ont partagé leurs idées au sujet de la sylviculture d'adaptation et de son intégration à la pratique en tenant compte des facteurs organisationnels et humains. Plus particulièrement, ils ont énuméré des défis de l'adaptation dans la pratique et lancé des appels à la collaboration. Ils ont fait ressortir comment la complexité du processus d'aménagement forestier nécessite une meilleure communication entre le milieu de la recherche et le milieu professionnel, un des défis auquel s'attaquait justement ce colloque.

Nous espérons que l'échange de connaissances et les réflexions des participants ainsi que la synthèse des risques, des possibilités et des solutions perçus aideront le secteur forestier à mettre en place des politiques et des mesures d'adaptation aux changements climatiques pour l'aménagement des forêts du XXI ${ }^{\mathrm{e}}$ siècle. 


\section{Remerciements}

Nous remercions le comité organisateur du Carrefour Forêts 2019 qui a permis et facilité la tenue de ce colloque. En particulier, notre reconnaissance va à Marie-Ėve Roy, Luciana Perecin et Hélène Labbé, qui ont contribué directement au succès de l'activité. Nous remercions également les coauteurs et coauteures des présentations, de même que les participants et participantes à la séance de présentations éclair : Donald Blouin (CERFO), Émilie Champagne (MFFP-DRF), Caroline Gagné (UQAM), Jérôme Laganière (RNCan-SCF), Miguel Montoro Girona (Swedish Agricultural University, UQAC), Sergio Rossi (UQAC), Samuel Roy Proulx (UQAT), Rita Sousa-Silva (ISFORT, UQO) et Mariétou Diouf (ULCEF, MFFP) ainsi que leur coauteurs. Finalement, nous étendons nos remerciements à Édouard Thiffault qui a traduit certaines portions du texte original, ainsi que Denise Tousignant (MFFP) pour la révision linguistique de la version française du manuscrit.

\section{Références}

Allen, C.D., A.K. Macalady, H. Chenchouni, D. Bachelet, N. McDowell, M. Vennetier, T. Kitzberger, A. Rigling, D.D. Breshears, E.H. Hogg, P. Gonzalez, R. Fensham, Z. Zhang, J. Castro, N. Demidova, J.-H. Lim, G. Allard, S.W. Running, A. Semerci et N. Cobb. 2010. A global overview of drought and heat-induced tree mortality reveals emerging climate change risks for forests. For. Ecol. Manage. 259(4): 660-684. doi:10.1016/j.foreco.2009.09.001.

Ameztegui, A., K.A. Solarik, J.R. Parkins, D. Houle, C. Messier et D. Gravel. 2018. Perceptions of climate change across the Canadian forest sector: The key factors of institutional and geographical environment. PLoS ONE 13(6). doi:10.1371/journal.pone.0197689.

Aubin, I., L. Boisvert-Marsh, H. Kebli, D. McKenney, J. Pedlar, K. Lawrence, E.H. Hogg, Y. Boulanger, S. Gauthier et C. Ste-Marie. 2018. Tree vulnerability to climate change: improving exposurebased assessments using traits as indicators of sensitivity. Ecosphere 9(2): e02108. doi:10.1002/ecs2.2108.

Aubin, I., C.M. Garbe, S. Colombo, C.R. Drever, D.W. McKenney, C. Messier, J. Pedlar, M.A. Saner, L.V.M. Wellstead, R. Winder, E. Witten et C. Ste-Marie. 2011. Why we disagree about assisted migration: Ethical implications of a key debate regarding the future of Canada's forests. For. Chron. 87(6): 755-765. doi:10.5558/ tfc2011-092.

Benomar, L., J. Beaulieu, M.S. Lamhamedi, A. Rainville et M.-C. Lambert. 2018. Migration assistée : utilisation de données éco-physiologiques pour redéfinir les territoires d'utilisation des semences pour les vergers de $2^{\mathrm{e}}$ génération et raffiner les modèles de transfert de semences pour les vergers de $1^{\text {re }}$ génération. Livrable 1 : Actualisation des territoires d'utilisation des vergers de deuxième génération de l'épinette blanche au Québec. Rapport de projet.

Bergeron, Y. 2000. Species and stand dynamics in the mixed woods of Quebec's southern boreal forest. Ecology 81: 1500-1516.

Bissonnette, J.F., J. Dupras, F. Doyon, C. Chion et J. Tardif. 2017. Perceptions of small private forest owner's vulnerability and adaptive capacity to environmental disturbances and climate change: Views from a heterogeneous population in southern Quebec, Canada. Small-Scale For. 16(3): 367-393. doi:10.1007/s11842-0169361-y.

Böcher, M. et M. Krott. 2014. The RIU model as an analytical framework for scientific knowledge transfer: the case of the "decision support system forest and climate change". Biodiv. Conserv. 23(14): 3641-3656. doi:10.1007/s10531-014-0820-5.

Bonan, G.B. 2008. Forests and climate change: forcings, feedbacks, and the climate benefits of forests. Science 320(5882): 1444-1449. doi:10.1126/science.1155121.

Boucher, D., S. Gauthier, N. Thiffault, W. Marchand, M. Girardin et M. Urli. 2020. How climate change might affect tree regeneration following fire at northern latitudes: a review. New For. 51: 543-571. doi:10.1007/s11056-019-09745-6.

Brandt, J.P., M.D. Flannigan, D.G. Maynard, I.D. Thompson et W.J.A. Volney. 2013. An introduction to Canada's boreal zone: ecosystem processes, health, sustainability, and environmental issues. Environ. Rev. 21(4): 207-226. doi:10.1139/er-2013-0040.

Capstick, S., L. Whitmarsh, W. Poortinga, N. Pidgeon et P. Upham. 2015. International trends in public perceptions of climate change over the past quarter century. WIREs Clim. Change 6(1): 3561. doi:10.1002/wcc.321

Depardieu, C., M.P. Girardin, S. Nadeau, P.R. Lenz, J. Bousquet et N. Isabel. 2020. Adaptive genetic variation to drought in a widely distributed conifer suggests a potential for increasing forest resilience in a drying climate. New Phytol. 227(2): 427-439. doi:10.1111/nph.16551.

D’Orangeville, L., D. Houle, L. Duchesne, R.P. Phillips, Y. Bergeron et D. Kneeshaw. 2018. Beneficial effects of climate warming on boreal tree growth may be transitory. Nature Comm. 9(1): 3213. doi:10.1038/s41467-018-05705-4.

Flannigan, M.D., B.J Stocks et B.M. Wotton. 2000. Climate change and forest fires. Sci. Total Environ. 262(3): 221-229. doi:10.1016/S0048-9697(00)00524-6.

Frelich, L.E. 2002. Forest Dynamics and Disturbance Regimes: Studies from Temperate Evergreen-Deciduous Forests. Cambridge University Press, Cambridge.

Fridley, J.D. 2012. Extended leaf phenology and the autumn niche in deciduous forest invasions. Nature 485(7398): 359-362. doi:10.1038/nature11056.

Gauthier, S., P. Bernier, T. Kuuluvainen, A.Z. Shvidenko et D.G. Schepaschenko. 2015. Boreal forest health and global change. Science 349(6250): 819-822. doi:10.1126/science.aaa9092.

Gauthier, S., P. Bernier, P.J. Burton, J. Edwards, K. Isaac, N. Isabel, K. Jayen, H. Le Goff et E.A. Nelson. 2014. Climate change vulnerability and adaptation in the managed Canadian boreal forest. Environ. Rev. 22: 256-285.

Glick P., B.A. Stein et N.A. Edelson. 2011. Scanning the conservation horizon: A guide to climate change vulnerability assessment. National Wildlife Federation. www.fs.usda.gov

Handler, S., M.J. Duveneck, L. Iverson, E. Peters, R.M. Scheller, K.R. Wythers, L. Brandt, P. Butler, M. Janowiak et P.D. Shannon. 2014. Minnesota forest ecosystem vulnerability assessment and synthesis: A report from the Northwoods Climate Change Response Framework project. U.S. Department of Agriculture, Forest Service, Northern Research Station. Gen. Tech. Rep. NRS-133. Newtown Square, PA.

Iverson, L.R., A.M. Prasad, S.N. Matthews et M. Peters. 2008. Estimating potential habitat for 134 eastern US tree species under six climate scenarios. For. Ecol. Manage. 254(3): 390-406.

Janowiak, M.K., A.W. D'Amato, C.W. Swanston, L. Iverson, F.R. Thompson, W.D. Dijak, S. Matthews, M.P. Peters, A. Prasad, J.S. Fraser, L.A. Brandt, P. Butler-Leopold, S.D. Handler, P.D. Shannon, D. Burbank, J. Campbell, C. Cogbill, M.J. Duveneck, M.R. Emery, N. Fisichelli, J. Foster, J. Hushaw, L. Kenefic, A. Mahaffey, T.L. Morelli, N.J. Reo, P.G. Schaberg, K.R. Simmons, A. Weiskittel, S. Wilmot, D. Hollinger, E. Lane, L. Rustad et P. Templer. 2018. New England and northern New York forest ecosystem vulnerability assessment and synthesis: a report from the New England Climate Change Response Framework project. U.S. Department of Agriculture, Forest Service, Northern Research Station. Gen. Tech. Rep. NRS-173. Newtown Square, PA.

Klenk, N., B. Adams, G. Bull, J. Innes, S. Cohen et B. Larson. 2011. Climate change adaptation and sustainable forest management: A proposed reflexive research agenda. For. Chron. 87(3): 351357. doi:10.5558/tfc2011-025.

Labonté, J., G. Drolet, J.D. Sylvain, N. Thiffault, F. Hébert et F. Girard. 2020. Phenology-based mapping of an alien invasive species using time series of multispectral satellite data: A case-study with glossy buckthorn in Québec, Canada. Remote Sens. 12(6): 922. doi:10.3390/rs12060922. 
Lamhamedi, M.S., A. Rainville, L. Benomar, I. Villeneuve, J. Beaulieu, J. Bousquet, H.A. Margolis, J. DeBlois et M.-C. Lambert. 2017. L'écophysiologie, un atout pour réussir la migration assistée de sources génétiques d'épinette blanche. Direction de la recherche forestière, Ministère des Forêts, de la Faune et des Parcs du Québec. Avis de recherche forestière no 89. Québec, QC. Disponible à : https://mffp.gouv.qc.ca/publications/forets/connaissances/ recherche/Lamhamedi-Mohammed/Avis89.pdf

Lemprière, T.C., W.A. Kurz, E.H. Hogg, C. Schmoll, G.J. Rampley, D. Yemshanov, D.W. McKenney, R. Gilsenan, A. Beatch, D. Blain, J.S. Bhatti et E. Krcmar. 2013. Canadian boreal forests and climate change mitigation. Environ. Rev. 21(4): 293-321. doi:10.1139/er-2013-0039.

Lenz, P.R.N., S. Nadeau, M.J. Mottet, M. Perron, N. Isabel, J. Beaulieu et J. Bousquet. 2020. Multi-trait genomic selection for weevil resistance, growth, and wood quality in Norway spruce. Evol. Appl. 13(1): 76-94. doi:10.1111/eva.12823.

Messier, C., J. Bauhus, F. Doyon, F. Maure, R. Sousa-Silva, P. Nolet, M. Mina, N. Aquilué, M.-J. Fortin et K. Puettmann. 2019. The functional complex network approach to foster forest resilience to global changes. For. Ecosyst. 6: 16. doi:10.1186/s40663-019-0166-2. Millar, C.I., N.L. Stephenson et S.L. Stephens. 2007. Change and forests of the future: Managing in the face of uncertainty. Ecol. Appl. 17(8): 2145-2151.

Ministère des Forêts de la Faune et des Parcs 2019. Actes du Carrefour Forêts 2019 : Des connaissances à la création de valeur. Gouvernement du Québec, Québec, QC. Disponible à : https://mffp. gouv.qc.ca/documents/forets/recherche/Actes_Carrefour_Forets_ 2019.pdf

Morin, H., D. Laprise et Y. Bergeron. 1993. Chronology of spruce budworm outbreaks near Lake Duparquet, Abitibi region, Quebec. Can. J. For. Res. 23(8): 1497-1506. doi:10.1139/x93-189.

Morin, M.B., D. Kneeshaw, F. Doyon, H. Le Goff, P. Bernier, V. Yelle, A. Blondlot et D. Houle. 2015. Climate change and the forest sector: Perception of principal impacts and of potential options for adaptation. For. Chron. 91(4): 395-406. doi:10.5558/tfc2015-069.

Nagel, L.M., B.J. Palik, M.A. Battaglia, A.W. D'Amato, J.M. Guldin, C.W. Swanston, M.K. Janowiak, M.P. Powers, L.A. Joyce, C.I. Millar, D.L. Peterson, L.M. Ganio, C. Kirschbaum et M.R. Roske. 2017. Adaptive silviculture for climate change: A national experiment in manager-scientist partnerships to apply an adaptation framework. J. For. 115(3): 167-178. doi:10.5849/jof.16-039.

Niinemets, Ü. et F. Valladares. 2006. Tolerance to shade, drought, and waterlogging of temperate northern hemisphere trees and shrubs. Ecol. Monogr. 76(4): 521-547. doi:10.1890/0012-9615(2006) 076[0521:ttsdaw $] 2.0 . c 0 ; 2$.

Ontl, T.A., C. Swanston, L.A. Brandt, P.R. Butler, A.W. D'Amato, S.D. Handler, M.K. Janowiak et P.D. Shannon. 2018. Adaptation pathways: ecoregion and land ownership influences on climate adaptation decision-making in forest management. Clim. Change 146(1): 75-88. doi:10.1007/s10584-017-1983-3.

Pavy, N., M. Lamothe, B. Pelgas, F. Gagnon, I. Birol, J. Bohlmann, J. Mackay, N. Isabel et J. Bousquet. 2017. A high resolution reference genetic map positioning $8.8 \mathrm{~K}$ genes for the conifer white spruce: structural genomics implications and correspondence with physical distance. Plant J. 90: 189-203. doi:10.1111/tpj.13478

Périé, C., S. de Blois, M.-C. Lambert et N. Casajus. 2014. Effets anticipés des changements climatiques sur l'habitat des espèces arborescentes au Québec. Ministère des Ressources naturelles du Québec, Direction de la recherche forestière, Mémoire de recherche forestière $\mathrm{n}^{\mathrm{o}} 173.46 \mathrm{p}$. Disponible à : https://mffp.gouv.qc.ca/documents/forets/connaissances/recherche/Memoire173.pdf

Perron, M., P., Lenz, M., Desponts, M., M.-J., Mottet, N. Isabel, J. Beaulieu et J. Bousquet. 2020. À l'aube d'une révolution : décoder le génome des arbres pour mieux aborder l'avenir. Avis de recherche forestière $\mathrm{n}^{\mathrm{O}} 133$. Gouvernement du Québec, ministère des Forêts, de la Faune et des Parcs, Direction de la recherche forestière. 2 p. Disponible à : https://mffp.gouv.qc.ca/documents/forets/recherche/ ARF133.pdf
Prasad, A.M., L.R. Iverson, S. Matthews et M. Peters. 2007. A Climate Change Atlas for 134 Forest Tree Species of the Eastern United States [database]. https://www.nrs.fs.fed.us/atlas/tree, Northern Research Station, USDA Forest Service, Delaware, Ohio.

Prévost, M. et J. DeBlois. 2014. Shelterwood cutting to release coniferous advance growth and limit aspen sucker development in a boreal mixedwood stand. For. Ecol. Manage. 323: 148-157.

Price, D.T., R.I. Alfaro, K.J. Brown, M.D. Flannigan, R.A. Fleming, E.H. Hogg, M.P. Girardin, T. Lakusta, M. Johnston, D.W. McKenney, J.H. Pedlar, T. Stratton, R.N. Sturrock, I.D. Thompson, J.A. Trofymow et L.A. Venier. 2013. Anticipating the consequences of climate change for Canada's boreal forest ecosystems. Environ. Rev. 21(4): 322-365. doi:10.1139/er-2013-0042.

Puettmann, K.J. et C. Messier. 2019. Simple guidelines to prepare forests for global change: The dog and the frisbee. Northwest Sci. 93: 209-225.

Rainville, A., J. Beaulieu, L. Langevin, T. Logan et M.-C. Lambert. 2014. Prédire l'effet des changements climatiques sur le volume marchand des principales espèces résineuses plantées au Québec, grâce à la génétique forestière. Direction de la recherche forestière, Ministère des Forêts, de la Faune et des Parcs du Québec. Mémoire de recherche forestière $\mathrm{n}^{\circ} 174$. Québec, QC. Disponible à : https:// mffp.gouv.qc.ca/documents/forets/connaissances/recherche/Memo ire174.pdf

Raymond, P. et S. Bédard. 2017. The irregular shelterwood system as an alternative to clearcutting to achieve compositional and structural objectives in temperate mixedwood stands. For. Ecol. Manage. 398: 91-100. doi:10.1016/j.foreco.2017.04.042

Raymond, P., A.A. Royo, M. Prévost et D. Dumais. 2018. Assessing the single-tree and small group selection cutting system as intermediate disturbance to promote regeneration and diversity in temperate mixedwood stands. For. Ecol. Manage. 430: 21-32. doi:10.1016/j.foreco.2018.07.054.

Raymond, P., S. Bédard, V. Roy, C. Larouche et S. Tremblay. 2009. The irregular shelterwood system: review, classification, and potential application to forests affected by partial disturbances. J. For. 107(8): 405-413. doi:10.1093/jof/107.8.405.

Reich, P.B., K.M. Sendall, K. Rice, R.L. Rich, L. Roy, A. Stefanski, S.E. Hobbie et R.A. Montgomery. 2015. Geographic range predicts photosynthetic and growth response to warming in co-occurring tree species. Nature Clim. Change. 5(2): 148-152. doi:10.1038/nclimate2497.

Spathelf, P., A. Bolte et E. van der Maaten. 2015. Is Close-toNature Silviculture (CNS) an adequate concept to adapt forests to climate change? Landbauforsch 3/4(65): 161-170. doi:10.3220/ LBF1452526188000.

Ste-Marie, C., E.A. Nelson, A. Dabros et M.-E. Bonneau. 2011. Assisted migration: Introduction to a multifaceted concept. For. Chron. 87(6): 724-730. doi:10.5558/tfc2011-089.

Thiffault, N. et F. Hébert. 2017. Mechanical site preparation and nurse-plant facilitation for the restoration of subarctic forest ecosystems. Can. J. For. Res. 47(7): 926-934. doi:10.1139/cjfr-2016-0448.

Trumbore, S., P. Brando et H. Hartmann. 2015. Forest health and global change. Science 349(6250): 814-818. doi:10.1126/science. aac6759.

Urli, M., N. Thiffault, D. Houle, S. Gauthier et Y. Bergeron. 2020. Role of green alder in boreal conifer growth: competitor or facilitator? FACETS 5(1): 166-181. doi:10.1139/facets-2019-0064.

Webster, C.R. et N.R. Jensen. 2007. A shift in the gap dynamics of Betula alleghaniensis in response to single-tree selection. Can. J. For. Res. 37(3): 682-689. doi:10.1139/x06-267.

Williamson, T.B., J.R. Parkins et B.L. McFarlane. 2005. Perceptions of climate change risk to forest ecosystems and forest-based communities. For. Chron. 81(5): 710-716. doi:10.5558/tfc81710-5. Williamson, T.B., M.H. Johnston, H.W. Nelson et J.E. Edwards. 2019. Adapting to climate change in Canadian forest management: Past, present and future. For. Chron. 95(2): 76-90. doi:10.5558/ tfc2019-015. 